\title{
Surface Physics of Semiconducting Nanowires
}

\author{
Michele Amato \\ Institut d'Electronique Fondamentale, UMR8622, CNRS, Universitè Paris-Sud, 91405 \\ Orsay, France \\ 5 \\ Riccardo Rurali* \\ Institut de Ciència de Materiales de Barcelona (ICMAB-CSIC), Campus de Bellaterra \\ 08193 Bellaterra (Barcelona), Spain
}

\begin{abstract}
Semiconducting nanowires (NWs) are firm candidates for novel nanoelectronic devices and a fruitful playground for fundamental physics. Ultra-thin nanowires, with diameters below $10 \mathrm{~nm}$, presents exotic quantum effects due to the confinement of the wave functions, e.g. broadening of the electronic band-gap, deepening of the dopant states. However, although several reports of sub-10 nm wires exist to date, the most common NWs have diameters that range from 20 to $200 \mathrm{~nm}$, where these quantum effects are absent or play a very minor role. Yet, the research activity on this field is very intense and these materials still promise to provide an important paradigm shift for the design of emerging electronic devices and different kinds of applications. A legitimate question is then: what makes a nanowire different from bulk systems? The answer is certainly the large surface-to-volume ratio.

In this article we discuss the most salient features of surface physics and chemistry in group-IV semiconducting nanowires, focusing mostly on Si NWs. First we review the state-of-the-art of NW growth to achieve a smooth and controlled surface morphology. Next we discuss the importance of a proper surface passivation and its role on the NW electronic properties. Finally, stressing the importance of a large surface-to-volume ratio and emphasizing the fact that in a NW the surface is where most of the action takes place, we discuss molecular sensing and molecular doping.
\end{abstract}

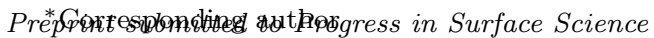

November 22, 2015

Email address: rrurali@icmab.es (Riccardo Rurali) 
Keywords: Semiconducting nanowires, surface physics, molecular doping, chemical sensors, biological detection

\section{Contents}

\begin{tabular}{lll}
\hline 1 & Introduction & 2
\end{tabular}

\begin{tabular}{|ll}
2 & Surface reconstruction and facet arrangement
\end{tabular}

\begin{tabular}{lll}
\hline 3 & Surface passivation & 11
\end{tabular}

$\begin{array}{lll}35 & \text { Surface physics and chemistry } & 15\end{array}$

4.1 Molecular doping . . . . . . . . . . . . . . . . . . 16

4.2 Chemical sensing with NWs . . . . . . . . . . . . . . . 19

$\begin{array}{lll}5 & \text { Conclusions } & 28\end{array}$

\section{Introduction}

40

Semiconducting nanowires (NWs) are rod-like pieces of semiconductor with a characteristic diameter in the nanometer scale. In a strongly confined regime nanowires exhibit an exotic behavior that reveals their quantum nature: the band-gap can be tuned [1, the density of states is quantized [2, luminescence can be largely increased $[3]$. As a matter of fact some of the early publications $[3$.

45 4, 5, 6, 7. even refer to them as quantum wires, to highlight the exquisitely quantum nature of these physical effects.

Nowadays, nanowires have become a credible platform on which to build the next generation of electron devices [8]. They are always semiconducting, because of their aspect ratio they can be simultaneously used as the active region of the device, e.g. the channel of a field-effect transistor, or to connect different devices [9], and -most importantly- they can be naturally integrated within the existing Si-based technology. Most of the applications where nanowires have shown superior performances, though, does not rely on those signatures of an 
underlying quantum world that captivated researchers in the beginning. Indeed, after the gold rush to the thinnest nanowire ever that yielded diameters in the $1-10 \mathrm{~nm}$ range 10, 11, 12, 13, 14, 15, 16, 17, 18, 19, 20, nanowires that are routinely grown today have a characteristic transverse size that typically lies in the 20-200 nm range where such quantum effects are at most negligible. Yet, nanowires keep on being an active fertile ground for fundamental research and advanced proof-of-concept devices, some of which outperform their conventional planar counterparts [21].

In this article we review the surface physics and chemistry of group-IV semiconducting nanowires -focusing on Si NWs. We argue that the high surface-tovolume ratio is one of the reasons for which nanowires are keeping the promise

65 to be nanomaterials that could drive innovation and that are an ideal test-bed for fundamental research. The paper is organized as follows: in Section 2 we review the factors that, at growth time, determine the crystallographic orientations of nanowires and, consequently, the facet arrangement; the chemical passivation of the nanowire surface and its effect on the nanowire properties are discussed in Section 3, molecular doping and sensing are critically examined in Section 4, where we review how molecular adsorbates can be used to tune the nanowire conductivity to engineer electronic devices (Section 4.1) or chemical sensors (Section 4.2); a brief overview of Surface Enhanced Raman Scattering (SERS) in nanowire is also given in the second part of Section 4.2 we give our 75 conclusions in Section 5.

\section{Surface reconstruction and facet arrangement}

The most fascinating peculiarity of Si NWs is the possibility to vary their properties by tailoring the size. As it has been demonstrated in many works (see Ref. 22] and references therein), the reduction of diameter in Si NWs can induce novel effects that have often a remarkable impact on both basic and applied research. The same happens with the study of NW surface structure. As a matter of fact, for such nanoscale systems, the traditional distinction between 
morphology - a term associated to a macroscopic property- and structure - a term which refers to a microscopic atomistic picture, focusing on the detailed atomic arrangement- cannot be applied anymore. In fact, due to the nanometric size, the two terms overlap and the atomistic structure may often determine the morphology of the wire [23]. When a nanowire is created the charge distribution as well as the atomic forces in play strongly change with respect to the bulk case [23]. Though it is always hard to find general rules for complex nanoscale processes, there are three main questions that one should address to understand the effect of the size on Si NWs structure: i) Which is the ground state crystal phase of Si NWs? The answer to this question is not obvious. In fact, while it is well known that bulk Si finds its lowest energetic configuration (at normal experimental conditions) in the diamond form, the organization of atoms at the nanoscale could in principle impose different atomic structures or novel phases (see for example the case of fullerene [24] and carbon nanotubes [25, 26]). ii) How do surafces reconstruct in a NW? As shown by experiments [27, the growth of a NW is a complex process, in which substrates, interfaces and thermodynamics of atoms play a crucial role. This can easily suggest that a simply scaling down properties of bulk surfaces could not be enough to understand surface structure in such kind of materials. iii) Can diameter affect also the growth direction of Si NWs? This question is intimately related to the previous two. As a matter of fact, an important consequence of a particular atomic configuration and shape is the associated crystallographic direction (if it is possible to define it).

Crystal phase of $\boldsymbol{S i} \boldsymbol{N W s}$ - The first experimental evidences of Si NWs growth rapidly indicated that these nanostructures are to a large extent crystalline. In particular it was found that they are rod-like structures constructed around a bulk Si single-crystalline core [22, 10, 11, 28, 29, 17, 15, 30]. This finding was mostly independent on the growth method, although it has been demonstrated that the quality of the crystal and the density of defects can depend on it [27]. All these experiments confirmed that, as can be intuitively expected, Si NWs crystallize in the diamond-like cubic structure as in their bulk 
counterpart (see Fig 1).

It is also worth highlighting the possibility, predicted in 2005 by Kagimura and co-workers [31] and recently demonstrated by experiments [32, 33, 34, 35, 36], to find evidences of wurtzite-type crystal in Si NWs. In group IV NWs the formation of different crystal phases is a rather common phenomenon and most nanowire growth schemes produces a rather random sequence of polytypes, with a high density of various stacking defects. However, recent results go even further by showing that the presence of hexagonal ordered phases as atomic stacking in cubic domains (homojunctions) can be controlled [37, 38].

The experimental characterization of Si NWs structure (a bulk diamond core which has a rod-like shape) was conducted in parallel with several theoretical works -mainly based on ab initio and semi-empirical methods- whose main objective was to theoretically determine the ground state phase of this class of nanostructures. In 2005 Kagimura and co-workers 31] studied by means of Density Functional Theory (DFT) calculations the structural stability of three different types of phases for Si NWs (with diameter smaller than $2 \mathrm{~nm}$ ): diamond-type, fullerene-like and high-density structures (like simple cubic or simple hexagonal lattice). Their results showed that, at subnanometer scale, though the diamond structure is favored, all the other considered phases were quite close in energy, pointing out the central role of surface effects. Similar studies were conducted by Ponomareva et al. [39, 40] who, by employing generalized Tight-Binding Molecular Dynamics investigated the stability of diamond, clathrate or polycrystalline phase in Si NWs with diameter between 1 and $5 \mathrm{~nm}$. While the instability of the polycrystalline phase was clearly assessed, the authors found the total energies of diamond and clathrate Si NWs comparable, but with the diamond phase still favored, i.e. more stable.

Shape and surface reconstruction - Once it has been established that the crystal phase of Si NWs is at a large extent diamond-cubic type, one would like to be able to determine the shape of its cross section and the atomic rearrangement of the surface. As previously pointed out, at nanoscale the morphology of the material and its atomic structure almost coincide and the role 
of edges can be crucial [41. The concept of free surface energy is hence fundamental in order to understand which will be the ground state shape of the NW. In particular, from a strictly theoretical point of view, the equilibrium shape of a solid, at fixed volume and chemical potential, is a complex polyhedron which minimizes the total free surface energy of the material (also known as Wulff plot [23]). The first experimental observations with transmission electron microscope (TEM) of NWs structures [10, 11, 28, 29, 17, 15, 30, evidenced that Si NWs surfaces (grown with both top-down and bottom-up approaches) are usually well facetted, with well defined lattice planes that determine specific cross sections. In particular, it has been demonstrated [17, 15] that the cross section of Si NWs grown along the $\langle 110\rangle$ crystallographic axis is hexagonal with well developed facets that correspond to the low free energy (111) and (100) planes (which is consistent with equilibrium shape calculated by the Wulff plot approach [42] and with the first principles calculations of Chan et al. [43]). On the other hand $\langle 111\rangle$ oriented NWs are presented as bounded (110) facets in the hexagonal shape 44. It is worth to note that both theoretical calculations and experiments indicate that hydrogen passivation of dangling bonds does not modify the morphology in a sizable way [45], but can induce sets of dimers with $\mathrm{SiH}$ species [46, 47].

The effect of diameter and edges on the surface reconstruction and on the thermodynamic equilibrium shape of NWs has also been the subject of an intense theoretical investigation from which the difficulty to figure out general rules at the ultimate nanoscale limit [22] emerged. In this context the contribution of Zhao and Yakobson has been very important 48]. They reformulated the Wulff approach for nanowires, sheding light on the fact that while in solids and nanoparticles the interaction between edges is usually negligible, the same does not happen in NWs for which the Wulff energy must include the term deriving from the matching of adjacent facets [48]. Within this formalism they found that the most stable NW at diameters less than $6 \mathrm{~nm}$ is a pentagonal cross section wire with a 5 -fold symmetry (that is polycrystalline) followed in energy by a single-crystal hexagonal wire. Although the predominance of pentagonal 
cross-section does not coincide with the trend observed in most of the experiments [10, 11, 28, 29, 17, 15, 30, evidences of a pentagonal cross section NWs reported in the experimental work of Furuya and colleagues [49] are worthy of note, although we are not aware of other reports of these structures.

In 1998 Ismail and Arias [50, by applying ab initio simulations to $\langle 100\rangle \mathrm{Si}$ NWs, showed that at small diameters NWs facets undergo a size-driven transition from a $2 \times 1$ to a $\mathrm{c}(2 \times 2)$ reconstruction. This transition results from the balance between bonding along the edges and the surface energy of the facets. In the same direction was the theoretical work of Cao et al. [51, who demonstrated that, driven by the combination of surface reconstruction and intercorner interaction, sharp corners structures can exist for NWs. The analysis of $\langle 100\rangle$ reconstructed NWs has been conducted by Rurali and Lorente [52, 53, 54], who, by employing DFT calculations, demonstrated that in absence of proper passivation the Si NW surface strongly reconstructs forming a series of buckled dimers noticeably affecting the electronic structure (a similar study for the $\langle 110\rangle$ was conducted by Singh et al. [55]). Moreover, the authors showed that for $\langle 100\rangle$ wires faceting favors the formation of (100) planes instead of (110) ones.

All the above considerations are referred to the primary faceting [56] which is the first level of the description of the surface faceting and that represents the overall shape of the wire cross section. The secondary faceting or more complex faceting phenomena (like sawtooth faceting [57]) are beyond the scope of this review and we refer the reader to more specific works (see for example Refs. [56, 57, 58, 59]).

Crystallographic orientation - As clearly pointed out by Schmidt et al. 27] the reduction of the nanowire diameter has two main effects on its structural properties which are strictly correlated: i) first, as is shown in the previous paragraphs, it influences the crystal phase, the final cross section shape and the surface reconstruction, ii) secondly, due to thermodynamic stability arguments, it can be responsible of a change of the crystallographic orientation during the growth process. Both these aspects are intimately related and have an enormous importance for the NWs physics because they will affect electronic 
and electrical properties [60, 27, 61, 62.

Assuming a vapor-liquid-solid (VLS) mechanism (the most common used method to obtain Si NWs [22]), the effect of the diameter on the growth direction of a NW can be easily explained by looking at the model presented in Figure 2

As is known, the VLS growth implies that gas silane molecules diffuse into the silicon-gold eutectic droplet, segregate at the liquid-solid interface and precipitate to build the Si NW crystal. Since the process takes place at the solidliquid interface, then the growth direction will be influenced by interface properties [63. Roughly speaking, the most preferred direction of growth will be the one that minimizes the total free energy of the interfaces. Among the others, there are two dominant energetic contributions that have to be considered if one approaches the study of the thermodynamics of this process: i) the first is the interfacial energy itself, which can be obtained by multiplying the interfacial surface $A$ for the corresponding liquid/solid interfacial tension $\sigma_{1 s}$, ii) the second is the silicon surface tension contribution from the edge of the interface (which is proportional to the circumference $L$, to the the surface silicon tension $\sigma_{s}$ and to the width of the interface on the silicon side $\Delta z$ (of the order of 1 $\mathrm{nm}$ [63]). If one introduces a geometrical parameter $a$ (function of $A, L$ and of the radius of the wire $r$ ), the specific free energy $f$ (per unit of circumference $L)$ of a $\langle 111\rangle$ oriented Si NW can be expressed as:

$$
f=\Delta z \cdot \sigma_{s}+a \cdot \sigma_{1 s} \cdot r
$$

An analogous expression can be derived for the interface energy of a $\langle 110\rangle$ oriented Si NW:

$$
f^{\prime}=\Delta z \cdot \sigma_{s}^{\prime}+a^{\prime} \cdot \sigma_{1 s}^{\prime} \cdot r
$$

where $a^{\prime}, \sigma_{s}^{\prime}$ and $\sigma_{1 s}^{\prime}$ are the corresponding parameters for a $\langle 110\rangle$ oriented system. All the quantities contained in both the equations can be evaluated by combining experimental results and theoretical approaches 63] (Young's equation and Wulff theorem). Their values are shown in Table 1. One can easily 


\begin{tabular}{cc}
\hline \hline$\langle 111\rangle$ orientation & $\langle 110\rangle$ orientation \\
\hline $\mathrm{a}=0.43$ & $a^{\prime}=0.39$ \\
$\sigma_{1 s}=0.62 \mathrm{~J} \cdot \mathrm{m}^{-2}$ & $\sigma_{1 s}^{\prime}=0.62 \mathrm{~J} \cdot \mathrm{m}^{-2}$ \\
$\sigma_{s}=1.53 \mathrm{~J} \cdot \mathrm{m}^{-2}$ & $\sigma_{s}^{\prime}=1.28 \mathrm{~J} \cdot \mathrm{m}^{-2}$ \\
\hline \hline
\end{tabular}

Table 1: Summary of parameters contained in Eq.1 and Eq. 2 [63.

observe that $\sigma_{s}>\sigma_{s}^{\prime}$ (due to the fact that a $\langle 111\rangle$ oriented nanowire presents an hexagonal shape with six (110) surface planes, while for a $\langle 110\rangle$ oriented NW

two of the six surface planes are (100) and four are (111) planes [63]) and that $a^{\prime} \sigma_{l s}^{\prime}>a \sigma_{s}$.

In Figure 3 the plot of the specific free energy for both $\langle 110\rangle$ and $\langle 111\rangle$ is reported.

Looking at the figure one can easily deduce that there is a critical value of the NW radius $r_{c}$ for which the curves intersect. This critical radius (that is of the order of $10 \mathrm{~nm}$, but that strongly depends on the ratio of interfacial and surface tensions) finds its analytic form in the following equation:

$$
r_{c}=\Delta z \frac{\sigma_{s}-\sigma_{s}^{\prime}}{a^{\prime} \sigma_{1 s}^{\prime}-a \sigma_{1 s}}
$$

This elegant model figures out which is the role of the diameter on the growth direction of a Si NW. For small diameters the most preferred crystallographic direction will be the $\langle 110\rangle$ because the system will have tendency to minimize the surface energy of the wire (see Eq. 1 and 2 and Table 1). On the other hand, at larger diameters, the main contribution to the free energy will be represented by the liquid-solid interfacial energy and so the preferential growth direction will be the $\langle 111\rangle$. The origin of this difference is due to the different scaling behavior of distinct energetic contributions: the one of the side faces is proportional to the diameter $d$ (through the circumference $L$ ) while the liquid-solid interface tension and the $\mathrm{Si}$ volume contribution scale with $d^{2}[27$. These results have been confirmed by the first-principles calculations of Rurali et al. [54] and Migas 
et al. [45. Furthermore, in 2006 Wang and co-workers 64 inserted these free of Schmidt et al. 63. Interestingly the $\langle 112\rangle$ growth direction which has been found for intermediate diameters (10 and $20 \mathrm{~nm}$ ) can be considered as 'transitional' direction between the $\langle 111\rangle$ and $\langle 110\rangle$ directions since the (112) plane is 
stepped plane between (111) and (110) planes 66, 67.

The strong dependence of VLS growth direction on the diameter in Si NWs does not set, however, which member of the crystallographic family will be favored. In fact even if a growth type is ensured $(\langle 110\rangle,\langle 111\rangle$ or $\langle 112\rangle)$ some wires might still choose one of the equivalent inclined directions of that crystallographic family (i.e. they will not necessarily grow perpendicularly to the substrate. This topic is beyond the scope of the present review and has been already carefully addressed in several works (see for example Ref. 68]).

\section{Surface passivation}

Unfortunately, when it comes to device engineering, the high chemical reacin non-negligible concentrations. For instance Baumer et al. 71 showed that $3.3 \times 10^{16} \mathrm{~cm}^{-3}$ dangling bonds or $\mathrm{P}_{b}$ centers are found at the oxide interface 
in a $10-15 \mathrm{~nm}$ Si NW.

A possible path to increase the quality of the Si NW-oxide interface is carrying out a $\mathrm{H}_{2}$ ambient annealing, which leads to dangling bond release from the wire surface and stress relaxation [72]. Dan et al. [73, on the other hand, reported in situ passivation with a thin a-Si layer, achieving an almost 2 orders of magnitude reduction of surface recombination, which results in an increase of the carrier diffusion length and lifetime by 1 and 2 orders of magnitude, respectively. Hydrogenated amorphous silicon (a-Si:H) was used by Yang et al. 74 . instead, resulting in an improved open-circuit voltage.

Ozone oxidation has also received some attention for the low temperature, defect-free oxidation of Si NWs. In bulk $\mathrm{Si}$ the $\mathrm{O}_{3}$-grown oxide exhibits improved interface and electrical characteristics over a conventionally dioxygen-grown oxide [75] and its use is started to be explored in nanowires, like in the work of Fukata et al. 20] (see Fig. 5). Interestingly, in this work the oxidation time is also used as a control parameter to obtain sub-10 nm Si NWs.

Due to the improved electrostatic control of the gate, the use of high- $\kappa$ oxides such as $\mathrm{HfO}_{2}$ has proven to play a crucial for the engineering of nanoelectronics devices with cutting edge performances, such as the transistor based on a SiGe core-shell NW reported by Xiang et al. 21. Surface passivation with a $\mathrm{HfO}_{2}$ layer results in a suppression of the non-radiative recombination rates and the sensing stability of Si NW arrays [76].

335

Thanks to its simplicity, reliability and stability, oxide passivation, in its various form, is the standard way to passivate surfaces in virtually all current nanowire applications. Alternative passivation strategies have been explored in an attempt to obtain ultra-clean surfaces, as interface defects are certainly the weakest point of oxidized surfaces. This goal can be fulfilled, at the expense of a long-term stability, with hydrogen passivation. Hydrogen passivation is conceptually the simplest way to passivate a Si surface, where each monovalent passivating agent - $\mathrm{a} \mathrm{H}$ atom- individually passivate each $\mathrm{Si}$ dangling bond. A simple way to obtain $\mathrm{H}$ passivation consists in the use of an aqueous HF etching of the oxidized silicon surfaces, which results in the removal of the surface oxide 
-the thinnest Si NW reported to date. This unprecedented resolution allowed them to unambiguously identify the hydrogen-terminated $\operatorname{Si}(111) 1 \times 1$ and $\mathrm{Si}(001) 1 \times 1$ surfaces corresponding to $\mathrm{SiH}_{3}$ on $\mathrm{Si}(111)$ and $\mathrm{SiH}_{2}$ on $\mathrm{Si}(001)$, respectively. more stable in air than their planar counterparts [15], they keep on being unsuitable for device fabrication where i) an oxide layer is often needed by the device design and ii) much longer stability lifetimes are requested. Yet, this approach has been followed by many groups working in fundamental studies of state-of-the-art characterization measurements -whether of structural [17, 57, optical [79] or electronic properties [80]- are in order.

An oxide-free route to nanowire passivation would be of great interest [1], because it would reduce the interface defect concentration, avoiding the related interface states that are highly detrimental of any the performances of any device with a large surface-to-volume ratio, such as those based on nanowires. As we said, freshly prepared H-terminated Si NWs have low surface recombination velocities, but are not stable in air 82] or in aqueous solutions 83 for more than a few hours. An idea to circumvent the poor stability of hydrogen passivation would be replacing the $\mathrm{Si}-\mathrm{H}$ bond with a stronger, more stable bond. The first important step in this direction was taken by Haick et al. [84, who reported $\mathrm{CH}_{3}$ passivated $\mathrm{Si}$ NWs with no intervening oxide, with good atmospheric stability, high conductance values, and low surface defect levels. These nanowires were then used for the fabrication of air-stable Si NW FETs. Just like in the 
probability of electron-hole recombination, thus increasing the carrier mobilities. Interestingly, the enhanced oxidation resistance under ambient conditions of methyl terminated Si NWs turns out to be even higher than equivalent 2D $\mathrm{Si}(100)$ and $\mathrm{Si}(111)$ surfaces with similar coverage [85]. These promising initial efforts were culminated by the work of Bashouti et al. [86, where chemical passivation of Si NWs with increasingly long and complex alkyl chains was demonstrated. Successful oxide-free passivated Si NWs were obtained using $\mathrm{CH}_{3}\left(\mathrm{CH}_{2}\right)_{(}(x-1)$ molecules, with $x=1-6$ (see Fig. 6). An instructive walkthrough on hydrosilylation, the insertion of an alkene or alkyne into a surface $\mathrm{Si}-\mathrm{H}$ bond to obtain such $\mathrm{Si}-\mathrm{C}$ bonds is given by Buriak et al. [87.

Some popular choices for planar solar cells, such as a-SiN ${ }_{x}: \mathrm{H}$ [88, 89, 90], $\mathrm{Al}_{2} \mathrm{O}_{3}$ [91, 92, 93. and the $\mathrm{SiO}_{2} / \mathrm{SiN}_{x}$ bilayer 94 have been recently explored for nanowires in an interesting comparative study by Dalmau Mallorquí et al. [95]. They achieved the highest efficiency with the $\mathrm{SiO}_{2} / \mathrm{SiN}_{x}$ stack.

The vast majority of theoretical studies consider hydrogen passivation, mostly because of its simplicity and because it is the passivation that requires the minimum number of atoms, thus making the calculation computationally lighter [22]. Although some studies have approached oxide passivation by terminating the dangling bonds with a OH group [96, 97, 98, or by bridging two Si atoms with 395 dangling bonds on a $\{100\}$ facet with an oxygen [99], a realistic description of a- $\mathrm{SiO}_{2}$ is much more challenging, as it requires accounting for the disordered structure of the amorphous oxide. Electronic structure and transport calculations at the first-principles level that include a quasi-amorphous $\mathrm{SiO}_{2}$ shell were reported by Koleini et al. [100. They combined classical and DFT molecular dynamics calculations to create the $\mathrm{Si} / \mathrm{SiO}_{x}$ core-shell structure and found that the conductance of the oxidized wires is lower with respect to the case of crystalline, H-passivated wires. They ascribed such a decrease to the structural distortions induced by the oxidation and to the presence of electronegative sites at the $\mathrm{Si} / \mathrm{SiO}_{x}$ interface [100].

405

A very appealing feature of nanostructures is that is often possible tuning their electronic properties by controlling some of the structural parameters or 
the composition. These effects have been widely discussed in nanowires, particularly for what concern the sensitivity of the electronic properties to changes in diameter, growth orientation, and composition [22, 101]. Several theoretical studies showed that a similar effect can be achieved by controlling the chemical properties of the passivating shell. Aradi et al. 97] compared $\mathrm{H}$ and $\mathrm{OH}$ passivation in $\langle 110\rangle$ and $\langle 112\rangle$ Si NWs, showing that a higher electronegativity of the passivating agent results in a narrower energy gap. Similar results were obtained by Nolan et al. 96, who also considered $\mathrm{NH}_{2}$, tracing the origin of the reduced band-gap to the hybridization between the Si $3 p$ and $\mathrm{O} / \mathrm{N} 2 \mathrm{p}$ states in the valence band edge. The work by $\mathrm{Ng}$ et al. 47] studied $\mathrm{OH}$ and $\mathrm{F}$ passivation, considering both full and partial substitution (in the case of partial substitution some of the dangling bonds are $\mathrm{H}$ terminated). Leu et al. [102], went a step further and, given a passivating species, also studied the dependence on the relative coverage, with respect to an all-H passivated nanowire. They studied $\langle 111\rangle,\langle 110\rangle,\langle 100\rangle$, and $\langle 112\rangle$ Si NWs with a passivating shell made of $\mathrm{A}_{x} \mathrm{H}_{1-x}$ atoms, where $\mathrm{A}$ is one of the halogens $\mathrm{Cl}, \mathrm{Br}$, and $\mathrm{I}$. The sensitivity to the chemical nature of the passivating elements is stronger, as expected, for thin nanowires and for coverages above $50 \%$. They found that the more weakly interacting species led to a larger band gap reduction, as the surface states were not pulled out of the midgap region by a strong binding.

\section{Surface physics and chemistry}

A surface is what makes a nanowire what it is: a confined quasi-one dimensional system whose properties differ form its bulk counterpart. In this section we will show that the surface is a privileged place for local chemistry, broadly speaking the adsorption of molecules, that can modify in a controlled way the electronic structure and the conductivity of a nanowire.

At the same time, however, even when no external chemical agent enters the picture, the mere existence of the surface alters the properties of the nanowire. 
are larger than in bulk as a result of the different way electrical charges are screened, even for thick wires where the effects of quantum confinement are weak. It has also been shown that the band offset at an axial interface is different than in planar interfaces and is strongly influenced by the nature of the surfaces and of the dipoles that develop there [104].

These topics have been reviewed elsewhere [22, 101] and we refer the interested reader to the original publications [103, 105, 104. In this section we focus on the role of adsorbates to tune the electrical conductivity of nanowires for applications in the field of sensor or to achieve electrical doping beyond the conventional use of ionized impurities.

\subsection{Molecular doping}

A semiconductor is a material which is about to conduct and where, therefore, conductivity can be tuned. The well-known way to perform this task is impurity doping [106]. Electronic device design, in the end, relies on the juxtaposition of regions of a semiconductor material with different doping characteristics.

Impurity doping in nanowires can be troublesome. An extensive discussion of the related issues can be found elsewhere [22] and here we limit ourselves to a brief summary of the reasons that can be detrimental to doping efficiency, without entering in details.

- Surface segregation-Impurities prefer surface and subsurface substitutional locations where they can more easily release the strain induced. There, they can be passivated and become electrically inactive [107, 108].

- Non-uniform dopant concentration-The competition between catalyzed and non-catalyzed dopant incorporation leads to radial [109] and axial [110] disuniformities in the impurity concentration.

- Quantum and dielectric confinement-As is well known, confinement pushes the otherwise shallow dopant levels down into the midgap region where they cannot be thermally activated [103, 111]. 
- Variability of the performances-Achieving typical impurity concenpurity scattering cross-section on their local environment is virtually eliminated, because less adsorption configurations exist and the associated scattering in the ballistic regime is almost negligible [120]. 
Several experimental results concerning the variation in the conductivity upon gas adsorption in mesoporous Si have been reported in the early 2000s. Porous Si, where electrochemical attack of a Si sample yields a disordered network of single-crystalline $\mathrm{Si}$ wires, is a very simple and cheap alternative to bottom-up Si nanowires and, due its sponge-like structure, is particularly suited for gas adsorption experiments. The donor character of $\mathrm{NH}_{3}$ was soon pointed out in many experimental studies [121, 122, 123, 124]. The doping mechanism was later revealed by first-principles calculations to simply rely on a shallow molecular orbital [125, 126, whose carriers can be thermally activated into the nanowire conduction band, analogously to textbook impurity doping. The same group worked intensively also on $\mathrm{NO}_{2}$ adsorption, which at first sight works as an acceptor [127, 128, 129, 130, 124], but in that case the reason for the observed increase in the conductivity was not as clear as it was with ammonia. It turns out that $\mathrm{NO}_{2}$ is not genuinely doping the semiconductor, but rather reactivates sub-surface passivated B atoms that remain buried, next to the surface, as a byproduct of the etching process [131. Therefore, $\mathrm{NO}_{2}$ is electronegative enough to take the electron of the surface dangling-bond, reactivating the passive $\mathrm{B}$, but not enough to create a hole in the valence band [125].

A more recent study focuses on the different mechanisms behind doping of Si nanowires upon molecular adsorption of two paradigmatic Lewis bases: pyridine and ethanol. Pyridine injects carriers from a shallow molecular level similarly to $\mathrm{NH}_{3}$. Ethanol, on the other hand, alters the dielectric screening conditions, allowing the reactivation of preexisting electrically passive impurities, just like $\mathrm{NO}_{2}$ does with passive sub-surface B atoms. A similar case was discussed by Timoshenko et al. [132, who used a simple model based on dielectric mismatch to show that the activation energy of a boron acceptor decreases from 105 to $30 \mathrm{meV}$ when the media dielectric constant increases from 1 (vacuum) to 25 (ethanol). Therefore, pyridine and similar molecules, such as ammonia and triethylamine ammonia behave like a conventional $n$-type dopant, inducing a free carrier concentration proportional to the number of adsorbed molecules. Ethanol (acetone or water) can act indirectly as a donor or an acceptor. The 
adsorption depend on the type and concentration of the impurities that are reactivated.

A remarkable demonstration of surface doping was also provided by Guo et al. [133], who reported hole and electron doping as a results of the adsorption of $\mathrm{H}_{2} \mathrm{O}$ and $\mathrm{NH}_{3}$ molecules and discussed the role of H-termination of the surface dangling bonds on the NW carrier concentration. The same group extended this study one year later, proposing an electron-transfer-dominated surface doping model to rationalize their results with Si NWs, e.g. p-type doping of H-passivated wires, sensitivity to the $\mathrm{pH}$ value of an adsorbed aqueous layer [134.

\subsection{Chemical sensing with $N W s$}

The extraordinary potential of Si NWs surface properties has been concreted in a lot of technological applications in which these nanostructures act as backbones for sensing devices. In its most general definition a sensor is a device that converts a detection event or a change in the environment into a measurable signal. Si NWs, pristine or specifically functionalized, can act as sensing device or components for the transduction of biological and chemical quantities into physical signals, mostly electrical or optical. As clearly explained in Ref. [135, 136, 137], traditional microelectronic sensors, among the other qualities they must possess -small dimension, low cost, low power consumption and compatibility with Si microelectronics- can be characterized by three main factors: i) the receptor function, ii) the transducer function and iii) the utility [135]. The first factor is related to the capability of the sensor to interact with the biological or chemical species to be detected. The transducer function is instead related to the easiness of the signal conversion, i.e. how the sensor material can convert the external signal into a variation of its intrinsic properties. The utility function takes usually into account kinetic effects related to the accessibility of molecular or gas species into the sensor surface. Interestingly, since the beginning of 90 s several experimental studies (see for example 
Ref. [138, 139, 140]) demonstrated that all the sensor performances (in particular the sensitivity, that is usually defined as the ratio between a detectable output signal to the input parameter) can be drastically improved by reducing the characteristic size of its component materials. As a consequence, research on materials for sensors has been focused on finding original ways to produce novel sensors based on nanostructures and nanomaterials.

Today Si NWs represent a viable solution to realize high-performance chemical and biological sensors due their unique properties and their potential for fabrication into high density nanoscale devices with low power consumption [141. In the last years this topic has been the subject of an intense research work 141, 142, 143, 144, 145, 146, 147, 148, 135, 149] whose main directions are not easy to be defined. However, the reasons for this huge interest and for the predominance of Si NWs in this field with respect to other nanostructures can be classified as follows: i) their large surface-to-volume ratio and their nano-size of the order of the Debye length. As pointed out in the previous sections, NWs have two dimensions that are usually less than $25 \mathrm{~nm}$. This makes surface effects dominate and induces a strong increase in the sensitivity of the material, i.e. in its capacity to convert the interaction with the biological or chemical species into a variation of its electronic/optical properties, ii) their excellent structural properties and their easiness of fabrication. Si NWs can be fabricated with a lot of different large scale techniques which always produce high crystalline, stable and reproducible semiconductor materials (in contrast to carbon nanotubes) that can be easily integrated into current Si technology, iii) the possibility to easily functionalize their oxide surface. As discussed in Section 3 . functionalization is an important aspect of Si NWs sensing as chemical or biological modification is often a fundamental requirement for their operation and biocompatibility [141, 142, 143, 144, 145, 146, 150]. Depending on the working principle of the sensor, metallic, covalent or non-covalent functionalization of $\mathrm{Si}$ NWs [141, 142, 151] have been proposed always assuming particular importance for the efficiency of the device, iv) the cylindrical 1D geometry that together with the small size induces electrical and optical properties that can be strongly 
influenced by minor perturbations. Indeed compared to $2 \mathrm{D}$ films in which the electrostatic changes due to the detection stay localized on the surface of the device, in Si NWs the modification of the surface potential creates an accumulation or depletion charge space region that covers also the bulk of wire (due to the small cross section area).

All things considered, it is clear that Si NWs can provide novel and unexplored sensing modalities. High label-free detection sensitivities of biomolecules, single molecules detection as well as chemical detection of various compounds have been reported [141, 142, 143, 144, 145, 146, 147, 148, 135, 152, 153, 154, 155. 156, 157. Although, due to the huge amount of articles, it is beyond the scope of this review to describe in detail all the specific works, a simple and straightforward distinction among Si NWs sensing devices can be done by classifying them on the basis of their working principle.

Electrochemical sensors - The basic physical mechanism governing the working principle of electrochemical Si NW based sensors is a standard fieldeffect as employed in a conventional Field Effect Transistor (FET). As is well known, the mode of operation of a classical planar FET is based on the variation of conductance of the semiconductor (usually p-type silicon) between source and drain by a third gate capacitively coupled by a dielectric layer [158, 144] as illustrated in Fig. 7. This operation concept made such kind of devices ideally suited for the detection of chemical and biological species, as demonstrated by the development of ion sensitive field-effect transistor (ISFET) [159]. Indeed the electric field induced by the binding of the chemical or biological species to the gate dielectric changes the conductance of the Si channel and provides the switching mode operation. However, despite its great potential, this approach was rapidly abandoned because of its low sensitivity.

On the other hand, the same underlying mechanism can be successfully reproduced by developing Si NWs based FETs [160, as illustrated in Fig. 8 .

Through the exploitation of the excellent electrical performances character615 istics of Si NWs FETs [161, 162, 163, 164, 165, 160, 166, in 2001 the Lieber group paved the way to this new research line by reporting the detection of 
different chemical and biological species in solution with extremely high sensitivities. This novel device concept (first realized with carbon nanotubes [167]) is simpler with respect to ISFETs because it is the nanowire itself that can act as conductive channel [147]. By looking at the scheme of Fig. 8 it is clear that the increased surface charge sensing effect occurs because of the reduced dimensions of the wire (that can be as small as 2-3 nm) that generate a large surface/volume ratio 168 . When a molecule or biomolecule links to the NW surface, depending on the surface orientation [169, it causes a variation of the surface potential which induces an accumulation or depletion of charges. This charge spatial variation, due to the reduced size of the system, directly affects the bulk properties of the wire, such as its electronic structure and its electrical conductivity. For example, in a p-type Si NWs, positive charge carriers on the surface can cause an electron accumulation in the bulk region of the wire hence reducing the electrical current between source and drain. On the other hand, negative charges on the surface can induce hole accumulation [147, 135]. The described physics can be analitycally converted in the following formula [135, 152]:

$$
\frac{\Delta G}{G}=\frac{\Delta n_{s}}{n_{0}}
$$

in which the variation of conductance before and upon molecular binding on the surface $(\Delta G)$ is proportional to the variation in charge carrier concentration due to the surface carrier variation $\left(\Delta n_{s}\right)$. Noticeably, it has been demonstrated that reducing the Si NW cross section from $200 \mathrm{~nm}$ to $50 \mathrm{~nm}$ can give a twentyfold increase of device sensitivity [170. However, a word of caution is in order here, because several works [171, 172] have also shown that the most appropriate way to improve device performances, and hence sensitivity, is to optimize the signal-to-noise ratio relative to the size and shape of the sensor.

As mentioned before, the first experimental reports of Si NWs FETs for biological and chemical detection dates back to the work of the Lieber's group in 2001 [143]. By functionalizing B-doped Si NWs surface with 3-aminopropyltriethoxysilane (APTES), antigen, biotin and calmodulin, high sensitivity detection (down to 

tively was reported [143, 135]. The underlying concept of these experiments relies on the surface functionalization and can be pointed out by describing the case of $\mathrm{pH}$ sensing. The functionalization of the oxide Si NW surface with APTES can serve as an optimal substrate for reaction of protonation and deprotonation by exploiting the surface NW potential changes before and upon chemical detection, as illustrated in Fig. 9(A).

The APTES terminating groups $-\mathrm{NH}_{2}$ and $\mathrm{SiOH}-$ have different dissociation constants and can switch the charge accumulation in the NW when the proton concentration is changed. In particular, at low $\mathrm{pH}$, the $\mathrm{NH}_{2}$ group is protonated to $\mathrm{NH}_{3}^{+}$and acts as a positive gate which depletes hole carriers in the p-type NW thus decreasing the conductance. On the other hand, at high pH levels, $\mathrm{SiOH}$ is deprotonated to $\mathrm{SiO}^{-}$and an increase in the conductance is observed. The resulting linear dependence [shown in Fig. 9(B)] observed was then theoretically explained by the model of Nair and Alam [173. All the cases studied in the work of Cui et al. 143 demonstrated that the sensing mechanism was the result of a field-effect equivalent to applying a voltage using a gate electrode [144] and that the functionalization was responsible for the high selectivity.

Following the same approach, by functionalizing Si NW surface with antibodies, the electrical selective detection of influenza A in solution was observed [174]. 65 Again, the basic operation mechanism of the device consists of a switch of the Si NW conductance by the binding and unbinding of the virus particle on the functionalized surface. Interestingly, in this work simultaneous detection was achieved by employing the excellent electrical performances of Si NWs arrays devices [175, 176, 177]. Moreover, in 2004 the use of Si NWs FET as sensors for single-stranded DNA was successfully demonstrated both for top-down [178] and bottom up Si NWs synthesis [179]. Modified Si NWs surface [with appropriate DNA receptors as peptide nucleic acid or 3-mercaptopropyltrimethoxysilane (MPTMS)] can easily bind with DNA sequences inducing a variation in carrier concentration in Si NWs and thus resulting in the changes of the conductance. Si NW FETs were also employed to demonstrate ultra-sensitive detection 
of bacterial toxin [Staphylococcus aureus Enterotoxin B (SEB)] with detection limits in the range of 10-35 fM [180].

It is worthwhile noting that in all the above mentioned works the chemical or biological detection was achieved in aqueous solution, a process that can be critical because of the dependence of the Debye screening -i.e. charge carrier's net electrostatic effect in solution- on sensor response and performances [181.

Equally challenging, but probably less complex, is the use of Si NWs for gas sensing. In this direction, since 2003, noticeable results were obtained by S.T. Lee group (see for example Ref. [182]). By developing a novel method for Si NWs synthesis, the oxide-assisted growth, they were able to fabricate high purity Si NWs in large quantities. These NWs were then HF-etched and installed to set up the device shown in Fig. 10 put in a vacuum chamber.

After evacuating the chamber, the gas species are introduced and then the variation of resistance in the device is measured. The chemical sensitivity to $\mathrm{NH}_{3}$ and water vapor was reported. In particular they found that both $\mathrm{NH}_{3}$ and water vapor could act as a molecular chemical gate resulting in hole depletion and resistance reduction (see top panel of Fig. 11). This effect is absent when the oxide is not removed from the surface thus revealing the high reactivity nature of pristine Si NW surfaces.

The same type of Si NWs was used to build a solution glucose bio-sensor by employing COOH-functionalized Si NWs [183, 184]. The main result here was demonstrating that the sensitivity strongly increased (in the range of 0.1$0.15 \mathrm{mM}$ ) with the treatment of carboxyl group with respect to HF and pristine NWs thus improving the transduction ability.

SERS sensors - The Surface Enhanced Raman Scattering (SERS) is a molecular spectroscopy technique that has been gaining much momentum since the discover of its basic principle in 1973 [142, 185, 186, 187, 188. In particular it occupies a special role in the field of chemical and biological sensing since it can provide ultrahigh sensitivity and excellent performances even in aqueous environment [189, 190] as demonstrated for the detection of DNA, $\mathrm{pH}$ values, protein or other biological molecules [191]. In the last years with the aim of 
improving the performances of SERS based devices -trying to improve the poor stability, the low reproducibility and the lack of homogeneity- many research efforts have been conducted towards the fabrication of SERS sensors in which Si NWs represent one of the backbone constituents. In order to illustrate which is the physical reason of this novel and original research line, the underlying operation mode of a SERS device merits being briefly described.

As elegantly explained in Ref. [191, the SERS process not only involves interactions between light and molecules, but also between light and metal nanostuctures. The mechanism is described in Fig. 12(A): an incoming electromagnetic wave of frequency $\omega_{i n c}$ can excite delocalized electrons in the metal (plasma oscillations) at a resonance frequency that depends on the dielectric functions of the metal and of the surrounding medium [191]. The resonance phenomenon [called Localized Surface Plasmon Resonance (LSPR)] makes electrons of the metal oscillating thus inducing a local electrical dipole which itself behaves as a nanoantenna, emitting radiation at the frequency $\omega_{\text {inc }}$ (see Fig. 12(A)). On the other hand, in the same way, the local electric field can excite the molecule placed near the metal nanostructure by inducing a dipole. Depending on the vibration frequency $\omega_{v i b}$ of the molecule, Rayleigh, Stokes Raman and anti-Stokes quantum mechanical effects and concern, respectively, a decrease and an increase of the emitted frequency with respect to the frequency of the incident light. The Stokes Raman scattering (that for molecules in their ground state is much more favored with respect to the anti-Stokes one) can produce an outcoming field of frequency $\omega_{i n c}-\omega_{v i b}$ itself again exciting and enhancing the LSPR of the metal nanostructure (see Fig. 12(B)).

This electromagnetic enhancement is called Surface Enhanced Raman Scattering (SERS) and has an intensity that depends on both the incoming and out coming field [191. As pointed out by Lee and co-workers [192] there are two main effects contributing to the enhancement of the Raman signal: a purely electromagnetic effect associated with the plasmonic resonance on the metal surface and a chemical effect responsible of the scattering process of the incoming wave 
due to the interaction between the molecule and the metal. The amplification of the signal (by enhancement factor of $10^{12-14}$ ) enables the detection of chemical and biological species even down to the single molecule level [193, 190.

Recently, it has been demonstrated that the fabrication of hierarchical structures composed by metal nanoparticles (NPs) and large nanosized supports can be essential for the building of highly efficient SERS devices [189, 194, 195, 196. 197. In particular the use of Si NWs as Ag and Au NPs support has an enormous potential for the design of nanosensors with ultrahigh sensitivity and specificity. In contrast to the electrochemical devices described in the previous paragraph, in this case the Si NWs do not represent the active part of the device (that is instead composed by metal NPs), but they play, however, a crucial role in the detection process. The three reasons of the interest metal NPs on the Si NW surface which reduces their agglomeration and guarantees high sensitivity and potential for real applications [193]. Furthermore, due to impregnation of metal NPs at the surface of Si NW and to their resistance 
to aggregation process, such kind of substrates show a substantial stability in 189

A prominent contribution to the development of these novel devices has been made by the group of S.T. Lee and co-workers. In 2008 [192] they used large scale uniform Si NWs array functionalized with Ag NPs to detect mouse immunoglobulin $\mathrm{G}$ (mIgG) and goat-anti-mouse immunoglobulin G (gamIgG) gamIgG in this substrate can give structural dependent Raman bands while their complexes produce distinct Raman bands for intensity and positions. The same kind of substrate was also used to detect Rhodamine 6G, crystal violet and nicotine 200] and, again, in 2010 201] to study the Raman spectra of five Sudan dyes in detail thanks to enhancement factors of $10^{8-10}$. Few years later [202] the same group reported the sensitive, specific and multiplex DNA detection on AgNPs@SiNWs SERS substrate with a concentration range of nearly 1 fM. More recently this type of substrate has been noticeably improved by increasing its reproducibility and selectivity [203].

AgNPs@SiNWs SERS single wire substrates were prepared by Han et al. 189] (see Fig. 14(a)) and employed for the detection of carbaryl residues on a cucumber surface and E. coli in drinking water. The latter experiment is presented in Fig 14 [panels (b) and (c)] from which the presence of carbaryl can be easily recognized looking at the peak at $1378 \mathrm{~cm}^{-1}$ appearing after transferring the AgNPs@SiNWs onto the probed surface.

It is important to point out that the research activity in the field of Si NWs based SERS sensors is very vivid and wide so that it results difficult to cover all the aspects of the problem. We refer the reader to specific reviews for a more accurate and detailed discussion [142, 193, 204, 157].

Finally, it is worth mentioning another type of optical Si NW device which is based on the fluorescence properties of functionalized Si NWs. By covalently modifying the Si NW surface with fluorescent ligands it is in fact possible to realize optical sensors which have a high sensitivity and selectivity, as demonstrated in the case of $\mathrm{Cu}(\mathrm{II})$ [205, 206], DNA [207] and nitric oxide (NO) 208] 
search approach has not been followed yet as the previous two, thus we address the reader to a few specific works [205, 206, 157].

\section{Conclusions}

Semiconducting nanowires are playing an increasingly important role in

to gain a full understanding and control of the surface phenomena here reviewed. defining the new generation electron devices and their interaction with the existing Si-based microelectronics technology. While in the beginning what captivated researchers was the possibility to highlight quantum effects of these systems, slowly a rich, equally interesting physics taking place at the surface started to emerge. In this article we have reviewed the most salient features mately what makes them what they are. We have first reviewed the morphology of the surfaces, their faceting, and chemical passivation, then focusing on the most significant progresses in the field of molecular doping and sensing (a distinction sometimes fictitious). The future of semiconducting nanowires and the success of the applications based thereupon will critically depend on our ability

\section{Acknowledgements}

We acknowledge support under contracts Nos. FEDER-MAT2013-40581-P of the Ministerio de Economía y Competitividad (MINECO), grant 2014 SGR gain a full understanding and control of the surface phenomena here reviewed. 301 of the Generalitat de Catalunya. and the Nanodesign project "Nanoharvesting" funded by the IDEX Paris-Saclay (ANR-11-IDEX-0003-02). Support from $\Psi_{k}$ and European Science Foundation (ESF) is also greatly acknowledged.

[1] M. Bruno, M. Palummo, A. Marini, R. Del Sole, S. Ossicini, From si nanowires to porous silicon: The role of excitonic effects, Phys. Rev. Lett. 98 (3) (2007) 036807. 
[2] E. Halpern, A. Henning, H. Shtrikman, R. Rurali, X. Cartoixà, Y. Rosenwaks, Room temperature observation of quantum confinement in single inas nanowires, Nano Lett. 15 (1) (2015) 481-485.

[3] L. T. Canham, Silicon quantum wire array fabrication by electrochemical and chemical dissolution of wafers, Appl. Phys. Lett. 57 (10) (1990) 10461048.

[4] V. Lehmann, U. Gösele, Porous silicon formation: A quantum wire effect, Appl. Phys. Lett. 58 (8) (1991) 856-858.

[5] F. Buda, J. Kohanoff, M. Parrinello, Optical properties of porous silicon: A first-principles study, Phys. Rev. Lett. 69 (8) (1992) 1272-1275.

[6] A. J. Read, R. J. Needs, K. J. Nash, L. T. Canham, P. D. J. Calcott, A. Qteish, First-principles calculations of the electronic properties of silicon quantum wires, Phys. Rev. Lett. 69 (8) (1992) 1232-1235.

[7] R. J. Needs, S. Bhattacharjee, K. J. Nash, A. Qteish, A. J. Read, L. T. Canham, First-principles calculations of band-edge electronic states of silicon quantum wires, Phys. Rev. B 50 (19) (1994) 14223-14227.

[8] Y. Li, F. Qian, J. Xiang, C. M. Lieber, Nanowire electronic and optoelectronic devices, Materials Today 9 (10) (2006) 18-27.

[9] D. K. Ferry, Materials science: Nanowires in nanoelectronics, Science 319 (5863) (2008) 579-580.

[10] A. M. Morales, C. M. Lieber, A laser ablation method for the synthesis of crystalline semiconductor nanowires, Science 279 (5348) (1998) 208-211.

[11] J. D. Holmes, K. P. Johnston, R. C. Doty, B. A. Korgel, Control of thickness and orientation of solution-grown silicon nanowires, Science 850 287 (5457) (2000) 1471-1473. 
[12] Y. Cui, L. J. Lauhon, M. S. Gudiksen, J. Wang, C. M. Lieber, Diametercontrolled synthesis of single-crystal silicon nanowires, Appl. Phys. Lett. 78 (15) (2001) 2214-2216.

[13] N. Coleman, M. Morris, T. Spalding, J. Holmes, The formation of dimensionally ordered silicon nanowires within mesoporous silica, J. Am. Chem. Soc. 123 (1) (2001) 187-188.

[14] N. R. B. Coleman, N. O'Sullivan, K. M. Ryan, T. A. Crowley, M. A. Morris, T. R. Spalding, D. C. Steytler, J. D. Holmes, Synthesis and characterization of dimensionally ordered semiconductor nanowires within mesoporous silica, J. Am. Chem. Soc. 123 (29) (2001) 7010-7016.

[15] D. D. D. Ma, C. S. Lee, F. C. K. Au, S. Y. Tong, S. T. Lee, Small-diameter silicon nanowire surfaces, Science 299 (5614) (2003) 1874-1877.

[16] Y. Cui, Z. Zhong, D. Wang, W. Wang, C. Lieber, High performance silicon nanowire field effect transistors, Nano Lett. 3 (2) (2003) 149-152.

[17] Y. Wu, Y. Cui, L. Huynh, C. Barrelet, D. Bell, C. Lieber, Controlled growth and structures of molecular-scale silicon nanowires, Nano Lett. 4 (3) (2004) 433-436.

[18] Z. Zhong, Y. Fang, W. Lu, C. M. Lieber, Coherent single charge transport in molecular-scale silicon nanowires, Nano Lett. 5 (6) (2005) 1143-1146.

[19] P. De Padova, C. Quaresima, P. Perfetti, B. Olivieri, C. Leandri, B. Aufray, S. Vizzini, G. Le Lay, Growth of straight, atomically perfect, highly metallic silicon nanowires with chiral asymmetry, Nano Lett. 8 (1) (2008) 271-275.

[20] N. Fukata, J. Kaminaga, R. Takiguchi, R. Rurali, M. Dutta, K. Murakami, 875 Interaction of boron and phosphorus impurities in silicon nanowires during low-temperature ozone oxidation, J. Phys. Chem. C 117 (39) (2013) 20300-20307. 
[21] J. Xiang, W. Lu, Y. Hu, H. Yan, C. M. Lieber, Ge/si nanowire heterostructures as high-performance field-effect transistors, Nature 441 (25) (2006) 489-493.

[22] R. Rurali, Colloquium: Structural, electronic, and transport properties of silicon nanowires, Rev. Mod. Phys. 82 (1) (2010) 427-449.

[23] H. Lüth, Solid surfaces, interfaces and thin films, Vol. 4, Springer, 2001.

[24] R. Haufler, J. Conceicao, L. Chibante, Y. Chai, N. Byrne, S. Flanagan, M. Haley, S. C. O'Brien, C. Pan, et al., Efficient production of c60 (buckminsterfullerene), c60h36, and the solvated buckide ion, J. Phys. Chem. 94 (24) (1990) 8634-8636.

[25] A. Oberlin, M. Endo, T. Koyama, Filamentous growth of carbon through benzene decomposition, J. Cryst. Growth 32 (3) (1976) 335-349.

[26] S. Iijima, et al., Helical microtubules of graphitic carbon, Nature 354 (6348) (1991) 56-58.

[27] V. Schmidt, J. V. Wittemann, U. Gösele, Growth, thermodynamics, and electrical properties of silicon nanowires, Chem. Rev. 110 (1) (2010) 361.

[28] Y. F. Zhang, L. S. Liao, W. H. Chan, S. T. Lee, R. Sammynaiken, T. K. Sham, Electronic structure of silicon nanowires: A photoemission and x-ray absorption study, Phys. Rev. B 61 (12) (2000) 8298-8305.

[29] B. Teo, X. Sun, T. Hung, X. Meng, N. Wong, S. Lee, Precision-cut crystalline silicon nanodots and nanorods from nanowires and direct visualization of cross sections and growth orientations of silicon nanowires, Nano Lett. 3 (12) (2003) 1735-1737.

[30] X.-Y. Zhang, L.-D. Zhang, G.-W. Meng, G.-H. Li, N. Jin-Phillipp, F. Phillipp, Synthesis of ordered single crystal silicon nanowire arrays, Adv. Mat. 13 (16) (2001) 1238. 
[31] R. Kagimura, R. W. Nunes, H. Chacham, Structures of si and ge nanowires in the subnanometer range, Phys. Rev. Lett. 95 (11) (2005) 115502.

[32] J. Arbiol, B. Kalache, P. R. i Cabarrocas, J. R. Morante, A. F. i Morral, Influence of $\mathrm{cu}$ as a catalyst on the properties of silicon nanowires synthesized by the vapour-solid-solid mechanism, Nanotechnology 18 (30) (2007) 305606.

[33] R. E. Algra, M. Hocevar, M. A. Verheijen, I. Zardo, G. G. Immink, W. J. van Enckevort, G. Abstreiter, L. P. Kouwenhoven, E. Vlieg, E. P. Bakkers, Crystal structure transfer in core/shell nanowires, Nano Lett. 11 (4) (2011) 1690-1694.

[34] F. J. Lopez, U. Givan, J. G. Connell, L. J. Lauhon, Silicon nanowire polytypes: identification by raman spectroscopy, generation mechanism, and misfit strain in homostructures, ACS Nano 5 (11) (2011) 8958-8966.

[35] F. J. Lopez, E. R. Hemesath, L. J. Lauhon, Ordered stacking fault arrays in silicon nanowires, Nano Lett. 9 (7) (2009) 2774-2779.

[36] Y. Li, Z. Liu, X. Lu, Z. Su, Y. Wang, R. Liu, D. Wang, J. Jian, J. H. Lee, H. Wang, Q. Yu, J. Bao, Broadband infrared photoluminescence in silicon nanowires with high density stacking faults, Nanoscale 7 (2015) 1601-1605.

[37] L. Vincent, G. Patriarche, G. Hallais, C. Renard, C. Gardès, D. Troadec, D. Bouchier, Novel heterostructured ge nanowires based on polytype transformation, Nano Lett. 14 (8) (2014) 4828-4836.

[38] S. Biswas, J. Doherty, D. Majumdar, T. Ghoshal, K. Rahme, M. Conroy, A. Singha, M. A. Morris, J. D. Holmes, Diameter controlled germanium nanowires with lamellar twinning and polytypes, Chem. Mater.

[39] I. Ponomareva, M. Menon, E. Richter, A. N. Andriotis, Structural stability, electronic properties, and quantum conductivity of small-diameter silicon nanowires, Phys. Rev. B 74 (12) (2006) 125311. 
[40] I. Ponomareva, M. Menon, D. Srivastava, A. N. Andriotis, Structure, stability, and quantum conductivity of small diameter silicon nanowires, Phys. Rev. Lett. 95 (26) (2005) 265502.

[41] J. F. Justo, R. D. Menezes, L. V. C. Assali, Stability and plasticity of silicon nanowires: The role of wire perimeter, Phys. Rev. B 75 (4) (2007) 045303.

[42] S. Hong, Equilibrium shape of hydrogen-covered diamond and silicon surfaces, J. Korean Phys. Soc. 37 (2) (2000) 93-98.

[43] T.-L. Chan, C. Ciobanu, F.-C. Chuang, N. Lu, C.-Z. Wang, K.-M. Ho, Magic structures of h-passivated 110 silicon nanowires, Nano Lett. 6 (2) (2006) 277-281.

[44] F. Li, P. D. Nellist, D. J. Cockayne, Doping-dependent nanofaceting on silicon nanowire surfaces, Appl. Phys. Lett. 94 (26) (2009) 263111.

[45] D. Migas, V. Borisenko, The role of morphology in stability of si nanowires, J. Appl. Phys. 105 (10) (2009) 104316-104316.

[46] T. Vo, A. J. Williamson, G. Galli, First principles simulations of the structural and electronic properties of silicon nanowires, Phys. Rev. B 74 (4) (2006) 045116.

${ }_{950}$ [47] M.-F. Ng, L. Zhou, S.-W. Yang, L. Y. Sim, V. B. C. Tan, P. Wu, Theoretical investigation of silicon nanowires: Methodology, geometry, surface modification, and electrical conductivity using a multiscale approach, Phys. Rev. B 76 (15) (2007) 155435.

[48] Y. Zhao, B. I. Yakobson, What is the ground-state structure of the thinnest si nanowires?, Phys. Rev. Lett. 91 (3) (2003) 035501.

[49] M. Takeguchi, M. Tanaka, H. Yasuda, K. Furuya, Real-time highresolution transmission electron microscopy observation of the growth process of (001) surfaces on a nanometer-sized si multiply twinned particle, Surf. Sci. 493 (1) (2001) 414-419. 
[50] S. Ismail-Beigi, T. Arias, Edge-driven transition in the surface structure of nanoscale silicon, Phys. Rev. B 57 (19) (1998) 11923.

[51] J. Cao, X. Gong, J. Zhong, R. Wu, Sharp corners in the cross section of ultrathin si nanowires, Phys. Rev. Lett. 97 (13) (2006) 136105.

[52] R. Rurali, N. Lorente, Metallic and semimetallic silicon $\langle 100\rangle$ nanowires, Phys. Rev. Lett. 94 (2) (2005) 026805.

[53] R. Rurali, N. Lorente, On the properties of surface reconstructed silicon nanowires, Nanotechnology 16 (2005) S250-S253.

[54] R. Rurali, A. Poissier, N. Lorente, Size effects in surface-reconstructed $\langle 100\rangle$ and $\langle 110\rangle$, Phys. Rev. B 74 (16) (2006) 165324.

[55] A. Singh, V. Kumar, R. Note, Y. Kawazoe, Pristine semiconducting [110] silicon nanowires, Nano Lett. 5 (11) (2005) 2302-2305.

[56] F. Oehler, P. Gentile, T. Baron, P. Ferret, M. Den Hertog, J. Rouviere, The importance of the radial growth in the faceting of silicon nanowires, Nano Lett. 10 (7) (2010) 2335-2341.

[57] F. M. Ross, J. Tersoff, M. C. Reuter, Sawtooth faceting in silicon nanowires, Phys. Rev. Lett. 95 (14) (2005) 146104.

[58] F. M. Ross, Controlling nanowire structures through real time growth studies, Rep. Prog. Phys. 73 (11) (2010) 114501.

[59] T. Xu, J. P. Nys, A. Addad, O. I. Lebedev, A. Urbieta, B. Salhi, M. Berthe, B. Grandidier, D. Stiévenard, Faceted sidewalls of silicon nanowires: Auinduced structural reconstructions and electronic properties, Phys. Rev. B 81 (11) (2010) 115403.

[60] M.-F. Ng, L. Shen, L. Zhou, S.-W. Yang, V. B. Tan, Geometry dependent i- v characteristics of silicon nanowires, Nano Letters 8 (11) (2008) 36623667. 
[61] M. Luisier, A. Schenk, W. Fichtner, Atomistic treatment of interface roughness in si nanowire transistors with different channel orientations, Appl. Phys. Lett. 90 (10) (2007) 102103.

[62] N. Neophytou, S. G. Kim, G. Klimeck, H. Kosina, On the bandstructure velocity and ballistic current of ultra-narrow silicon nanowire transistors as a function of cross section size, orientation, and bias, J. Appl. Phys. 107 (11) (2010) 113701.

[63] V. Schmidt, S. Senz, U. Gosele, Diameter-dependent growth direction of epitaxial silicon nanowires, Nano Lett. 5 (5) (2005) 931-935.

[64] C. Wang, M. Hirano, H. Hosono, Origin of diameter-dependent growth direction of silicon nanowires, Nano Lett. 6 (7) (2006) 1552-1555.

[65] Y.-H. Huang, Competition between surface energy and interphase energy in transition region and diameter-dependent orientation of silicon nanowires, Appl. Surf. Sci. 255 (7) (2009) 4347-4350.

[66] W. Lu, C. M. Lieber, Semiconductor nanowires, J. Phys. D: Appl. Phys. 39 (21) (2006) R387-R406.

[67] T. Tan, S. Lee, U. Gösele, A model for growth directional features in silicon nanowires, Appl. Phys. A 74 (3) (2002) 423-432.

[68] H. Schmid, M. Björk, J. Knoch, H. Riel, W. Riess, P. Rice, T. Topuria, Patterned epitaxial vapor-liquid-solid growth of silicon nanowires on si (111) using silane, J. Appl. Phys. 103 (2) (2008) 024304.

[69] A. G. Aberle, Surface passivation of crystalline silicon solar cells: a review, Prog. Photovoltaics 8 (5) (2000) 473-487.

[70] E. H. Nicollian, Surface passivation of semiconductors, J. Vac. Sci. Tech1010 nol. 8 (5) (1971) S39-S49.

[71] A. Baumer, M. Stutzmann, M. S. Brandt, F. C. Au, S. T. Lee, Paramagnetic defects of silicon nanowires, Appl. Phys. Lett. 85 (6) (2004) 943-945. 
[72] B. S. Swain, S. S. Lee, S. H. Lee, B. P. Swain, N. M. Hwang, Effect of $\{$ H2 $\}$ ambient annealing on silicon nanowires prepared by atmospheric pressure chemical vapor deposition, Chem. Phys. Lett. 494 (4-6) (2010) 269-273.

[73] Y. Dan, K. Seo, K. Takei, J. H. Meza, A. Javey, K. B. Crozier, Dramatic reduction of surface recombination by in situ surface passivation of silicon nanowires, Nano Lett. 11 (6) (2011) 2527-2532.

[74] P. Yang, X. Zeng, X. Xie, X. Zhang, H. Li, Z. Wang, Improved opencircuit voltage of silicon nanowires solar cells by surface passivation, RSC Adv. 3 (2013) 24971-24974.

[75] C. K. Fink, K. Nakamura, S. Ichimura, S. J. Jenkins, Silicon oxidation by ozone, J. Phys.: Cond. Matter 21 (18) (2009) 183001.

[76] L. Gan, H. He, L. Sun, Z. Ye, Improved photoluminescence and sensing stability of porous silicon nanowires by surface passivation, Phys. Chem. Chem. Phys. 16 (2014) 890-894.

[77] G. S. Higashi, Y. J. Chabal, G. W. Trucks, K. Raghavachari, Ideal hydrogen termination of the si(111) surface, Appl. Phys. Lett. 56 (7) (1990) 656-658.

[78] Y. Morita, K. Miki, H. Tokumoto, Direct observation of sih3 on a 1si(111) surface by scanning tunneling microscopy, Appl. Phys. Lett. 59 (11) (1991) 1347-1349.

[79] A. Guichard, D. Barsic, S. Sharma, T. Kamins, M. Brongersma, Tunable light emission from quantum-confined excitons in $\mathrm{TiSi}_{2}$-catalyzed silicon nanowires, Nano Lett. 6 (9) (2006) 2140-2144.

[80] R. He, P. Yang, Giant piezoresistance effect in silicon nanowires, Nat. Nanotechnol. 1 (2006) 42-46.

[81] M. Y. Bashouti, K. Sardashti, S. W. Schmitt, M. Pietsch, J. Ristein, H. Haick, S. H. Christiansen, Oxide-free hybrid silicon nanowires: From 

39-60.

[82] M. Bashouti, K. Sardashti, J. Ristein, S. Christiansen, Kinetic study of h-terminated silicon nanowires oxidation in very first stages, Nanoscale Res. Lett. 8 (1) (2013) 41.

[83] C. Peng, J. Gao, S. Wang, X. Zhang, X. Zhang, X. Sun, Stability of hydrogen-terminated surfaces of silicon nanowires in aqueous solutions, J. Phys. Chem. C 115 (10) (2011) 3866-3871.

[84] H. Haick, P. T. Hurley, A. I. Hochbaum, P. Yang, N. S. Lewis, Electrical characteristics and chemical stability of non-oxidized, methyl-terminated silicon nanowires, J. Am. Chem. Soc. 128 (28) (2006) 8990-8991.

[85] M. Y. Bashouti, Y. Paska, S. R. Puniredd, T. Stelzner, S. Christiansen, H. Haick, Silicon nanowires terminated with methyl functionalities exhibit stronger si-c bonds than equivalent 2d surfaces, Phys. Chem. Chem. Phys. 11 (2009) 3845-3848.

[86] M. Y. Bashouti, T. Stelzner, A. Berger, S. Christiansen, H. Haick, Chemical passivation of silicon nanowires with c1-c6 alkyl chains through covalent si-c bonds, J. Phys. Chem. C 112 (49) (2008) 19168-19172.

[87] J. M. Buriak, Illuminating silicon surface hydrosilylation: An unexpected plurality of mechanisms, Chem. Mater. 26 (1) (2014) 763-772. recombination velocities on $1 \mathrm{~cm}$ p-silicon using remote plasma silicon nitride passivation, Appl. Phys. Lett. 68 (9) (1996) 1232-1234.

[89] A. G. Aberle, R. Hezel, Progress in low-temperature surface passivation of silicon solar cells using remote-plasma silicon nitride, Prog. Photovoltaics 5 (1) (1997) 29-50. 
[90] S. De Wolf, G. Agostinelli, G. Beaucarne, P. Vitanov, Influence of stoichiometry of direct plasma-enhanced chemical vapor deposited sinx films and silicon substrate surface roughness on surface passivation, J. Appl. Phys. 97 (6) (2005) 063303.

1070 [91] G. Agostinelli, A. Delabie, P. Vitanov, Z. Alexieva, H. Dekkers, S. D. Wolf, G. Beaucarne, Very low surface recombination velocities on p-type silicon wafers passivated with a dielectric with fixed negative charge, Sol. Energy Mater. Sol. Cells 90 (18-19) (2006) 3438 - 3443.

[92] B. Hoex, S. B. S. Heil, E. Langereis, M. C. M. van de Sanden, W. M. M. Kessels, Ultralow surface recombination of c-si substrates passivated by plasma-assisted atomic layer deposited al2o3, Appl. Phys. Lett. 89 (4) (2006) 042112.

[93] B. Hoex, J. J. H. Gielis, M. C. M. van de Sanden, W. M. M. Kessels, On the c-si surface passivation mechanism by the negative-charge-dielectric al2o3, J. Appl. Phys. 104 (11) (2008) 113703.

[94] Z. Chen, P. Sana, J. Salami, A. Rohatgi, A novel and effective pecvd sio2/sin antireflection coating for si solar cells, Electron Devices, IEEE Transactions on 40 (6) (1993) 1161-1165.

[95] A. D. Mallorquí, E. Alarcón-Lladó, I. Mundet, A. Kiani, B. Demaurex, S. De Wolf, A. Menzel, M. Zacharias, A. Fontcuberta i Morral, Field-effect passivation on silicon nanowire solar cells, Nano Res. (2014) 1-9.

[96] M. Nolan, S. O'Callaghan, G. Fagas, J. Greer, T. Frauenheim, Silicon nanowire band gap modification, Nano Lett. 7 (1) (2007) 34-38.

[97] B. Aradi, L. E. Ramos, P. Deak, T. Kohler, F. Bechstedt, R. Q. Zhang, 1090 T. Frauenheim, Theoretical study of the chemical gap tuning in silicon nanowires, Phys. Rev. B 76 (3) (2007) 035305.

[98] G. Fagas, J. C. Greer, Ballistic conductance in oxidized si nanowires, Nano Lett. 9 (5) (2009) 1856-1860. 
[99] P. V. Avramov, A. A. Kuzubov, A. S. Fedorov, P. B. Sorokin, F. N.

M. Koleini, L. Colombi Ciacchi, M. V. Fernández-Serra, Electronic transport in natively oxidized silicon nanowires, ACS Nano 5 (4) (2011) 2839-

[101] M. Amato, M. Palummo, R. Rurali, S. Ossicini, Silicon-germanium nanowires: Chemistry and physics in play, from basic principles to advanced applications, Chem. Rev. 114 (2) (2014) 1371-1412.

[102] P. W. Leu, B. Shan, K. Cho, Surface chemical control of the electronic

Tomilin, Y. Maeda, Density-functional theory study of the electronic structure of thin $\mathrm{Si} / \mathrm{Sio}_{2}$ quantum nanodots and nanowires, Phys. Rev. B 75 (2007) 205427. 2846. structure of silicon nanowires: Density functional calculations, Phys. Rev. B 73 (19) (2006) 195320.

[103] M. Diarra, Y.-M. Niquet, C. Delerue, G. Allan, Ionization energy of donor and acceptor impurities in semiconductor nanowires: Importance of dielectric confinement, Phys. Rev. B 75 (4) (2007) 045301.

[104] Y.-M. Niquet, C. Delerue, Band offsets, wells, and barriers at nanoscale semiconductor heterojunctions, Phys. Rev. B 84 (2011) 075478.

[105] M. Diarra, C. Delerue, Y.-M. Niquet, G. Allan, Screening and polaronic effects induced by a metallic gate and a surrounding oxide on donor and acceptor impurities in silicon nanowires, J. Appl. Phys. 103 (7) (2008) 073703.

[106] R. S. Muller, T. I. Kamins, Device Electronics for Integrated Circuits, John Wiley \& Sons, Hoboken, NJ, 1986.

[107] M. V. Fernández-Serra, C. Adessi, X. Blase, Surface segregation and backscattering in doped silicon nanowires, Phys. Rev. Lett. 96 (16) (2006) 166805. 
[108] H. Peelaers, B. Partoens, F. Peeters, Formation and segregation energies of $\mathrm{b}$ and $\mathrm{p}$ doped and bp codoped silicon nanowires, Nano Lett. 6 (12) (2006) 2781-2784.

[109] D. E. Perea, E. R. Hemesath, E. J. Schwalbach, J. L. Lensch-Falk, P. W. Voorhees, L. J. Lauhon, Direct measurement of dopant distribution in an individual vapour-liquid-solid nanowire, Nature Nanotech. 4 (5) (2009) $315-319$.

[110] G. Imamura, T. Kawashima, M. Fujii, C. Nishimura, T. Saitoh, S. Hayashi, Distribution of active impurities in single silicon nanowires, Nano Lett. 8 (9) (2008) 2620-2624.

[111] M. T. Björk, H. Schmid, J. Knoch, H. Riel, W. Riess, Donor deactivation in silicon nanostructures, Nat. Nanotech. 4 (2) (2009) 103-107.

[112] F. Iori, S. Ossicini, R. Rurali, Conductance fluctuations in si nanowires studied from first-principles, J. Appl. Phys. 116 (7) (2014) 074303.

1135 [113] M.-V. Fernández-Serra, C. Adessi, X. Blase, Conductance, surface traps, and passivation in doped silicon nanowires, Nano Lett. 6 (12) (2006) 26742678.

[114] T. Markussen, R. Rurali, A.-P. Jauho, M. Brandbyge, Transport in silicon nanowires: role of radial dopant profile, J. Comput. Electron. 7 (2008) $324-327$.

[115] T. Markussen, R. Rurali, A.-P. Jauho, M. Brandbyge, Scaling theory put into practice: First-principles modeling of transport in doped silicon nanowires, Phys. Rev. Lett. 99 (7) (2007) 076803.

[116] R. Rurali, T. Markussen, J. Suñe, M. Brandbyge, A.-P. Jauho, Modeling transport in ultrathin si nanowires: Charged versus neutral impurities, Nano Lett. 8 (9) (2008) 2825-2828. 
[117] C.-Y. Moon, W.-J. Lee, K. J. Chang, Formation of dopant-pair defects and doping efficiency in b- and p-doped silicon nanowires, Nano Lett. 8 (10) (2008) 3086-3091.

[123] F. Geobaldo, P. Rivolo, G. P. Salvador, G. Amato, L. Boarino, E. Garrone, Free carriers reactivation on $\mathrm{p}+$-mesoporous silicon through ammonia adsorption: a ftir study, Sens. Actuators B 100 (1-2) (2004) 205-208.

[124] E. Garrone, F. Geobaldo, P. Rivolo, G. Amato, L. Boarino, M. Chiesa, 1170

[118] T. He, J. He, M. Lu, B. Chen, H. Pang, W. F. Reus, W. M. Nolte, D. P. Nackashi, P. D. Franzon, J. M. Tour, Controlled modulation of conductance in silicon devices by molecular monolayers, J. Am. Chem. Soc. 128 (45) (2006) 14537-14541.

[119] T. He, D. A. Corley, M. Lu, N. H. D. Spigna, J. He, D. P. Nackashi, P. D. Franzon, J. M. Tour, Controllable molecular modulation of conductivity in silicon-based devices, J. Am. Chem. Soc. 131 (29) (2009) 10023-10030.

[120] X. Blase, M.-V. Fernandez-Serra, Preserved conductance in covalently functionalized silicon nanowires, Phys. Rev. Lett. 100 (4) (2008) 046802.

[121] F. Geobaldo, P. Rivolo, M. Rocchia, A. M. Rossi, E. Garrone, Free carriers reactivation in mesoporous $\mathrm{p}+$-type silicon by ammonia condensation: an ftir study, Phys. Stat. Sol. (a) 197 (2) (2003) 458-461.

[122] M. Chiesa, G. Amato, L. Boarino, E. Garrone, F. Geobaldo, E. Giamello, Reversible insulator-to-metal transition in $\mathrm{p}^{+}$-type mesoporous silicon induced by the adsorption of ammonia, Angew. Chem. Int. Edit. 42 (41) (2003) 5032-5035.

E. Giamello, R. Gobetto, P. Ugliengo, A. Viale, A nanostructured porous silicon near insulator becomes either a p- or an n-type semiconductor upon gas adsorption, Adv. Mater. 17 (5) (2005) 528-531. 
[125] A. Miranda-Durán, X. Cartoixà, M. Cruz Irisson, R. Rurali, Molecular doping and subsurface dopant reactivation in si nanowires, Nano Lett.

[126] A. Miranda, X. Cartoixà, E. Canadell, R. Rurali, Nh3 molecular doping of silicon nanowires grown along the [112], [110], [001], and [111] orientations, Nanoscale Res. Lett. 7 (1) (2012) 308.

[127] L. Boarino, F. Geobaldo, S. Borini, A. M. Rossi, P. Rivolo, M. Rocchia,

[129] E. Garrone, S. Borini, P. Rivolo, L. Boarino, F. Geobaldo, G. Amato, Porous silicon in no2: A chemisorption mechanism for enhanced electrical conductivity, Phys. Stat. Sol. (a) 197 (1) (2003) 103-106.

[130] F. Geobaldo, P. Rivolo, S. Borini, L. Boarino, G. Amato, M. Chiesa, E. Garrone, Chemisorption of no2 at boron sites at the surface of nanostructured mesoporous silicon, J. Phys. Chem. B 108 (47) (2004) 1830618310.

[131] G. Polisski, D. Kovalev, G. Dollinger, T. Sulima, F. Koch, Boron in mesoporous si - where have all the carriers gone?, Physica B 273-274 (1999) 1195

[132] V. Y. Timoshenko, T. Dittrich, V. Lysenko, M. G. Lisachenko, F. Koch, Free charge carriers in mesoporous silicon, Phys. Rev. B 64 (8) (2001) 085314 . 
[133] C.-S. Guo, L.-B. Luo, G.-D. Yuan, X.-B. Yang, R.-Q. Zhang, W.-J. Zhang,

[141] A. K. Wanekaya, W. Chen, N. V. Myung, A. Mulchandani, Nanowirebased electrochemical biosensors, Electroanal. 18 (6) (2006) 533.

[142] Y. He, C. Fan, S.-T. Lee, Silicon nanostructures for bioapplications, Nano Today 5 (4) (2010) 282-295. 
[143] Y. Cui, Q. Wei, H. Park, C. M. Lieber, Nanowire nanosensors for highly

[144] F. Patolsky, C. M. Lieber, Nanowire nanosensors, Mater. today 8 (4) (2005) 20-28.

[145] B. He, T. J. Morrow, C. D. Keating, Nanowire sensors for multiplexed

[146] X.-J. Huang, Y.-K. Choi, Chemical sensors based on nanostructured materials, Sensor Actuat. B-Chem. 122 (2) (2007) 659-671.

[147] E. T. Carlen, A. van den Berg, Nanowire electrochemical sensors: can we live without labels?, Lab Chip 7 (1) (2007) 19-23.

[148] F. Patolsky, G. Zheng, C. M. Lieber, Nanowire-based biosensors, Anal. Chem. 78 (13) (2006) 4260-4269.

[149] S. Su, W. Wu, J. Gao, J. Lu, C. Fan, Nanomaterials-based sensors for applications in environmental monitoring, Journal of Materials Chemistry 22 (35) (2012) 18101-18110.

1240

[150] M. Amato, R. Rurali, Shell-thickness controlled semiconductor-metal transition in si-sic core-shell nanowires, Nano Lett. 15 (5) (2015) 34253430.

[151] R. Chen, D. Li, H. Hu, Y. Zhao, Y. Wang, N. Wong, S. Wang, Y. Zhang, J. Hu, Z. Shen, et al., Tailoring optical properties of silicon nanowires by au nanostructure decorations: enhanced raman scattering and photodetection, The Journal of Physical Chemistry C 116 (7) (2012) 4416-4422.

[152] X. Chen, C. K. Wong, C. A. Yuan, G. Zhang, Nanowire-based gas sensors, Sensors and Actuators B: Chemical 177 (2013) 178-195. 
[153] E. Stern, J. F. Klemic, D. A. Routenberg, P. N. Wyrembak, D. B. Turner-

[161] Y. Cui, X. Duan, J. Hu, C. M. Lieber, Doping and electrical transport in silicon nanowires, J. Phys. Chem. B 104 (22) (2000) 5213-5216.

[162] S.-W. Chung, J.-Y. Yu, J. R. Heath, Silicon nanowire devices, Appl. Phys. Lett. 76 (15) (2000) 2068-2070. 
[163] J.-Y. Yu, S.-W. Chung, J. R. Heath, Silicon nanowires: preparation, device fabrication, and transport properties, The Journal of Physical Chemistry B 104 (50) (2000) 11864-11870.

[164] Y. Cui, C. M. Lieber, Functional nanoscale electronic devices assembled using silicon nanowire building blocks, Science 291 (5505) (2001) 851-853.

[165] S. Jin, D. Whang, M. C. McAlpine, R. S. Friedman, Y. Wu, C. M. Lieber, Scalable interconnection and integration of nanowire devices without registration, Nano Letters 4 (5) (2004) 915-919.

[166] G. Zheng, W. Lu, S. Jin, C. M. Lieber, Synthesis and fabrication of high-performance n-type silicon nanowire transistors, Advanced Materials 16 (21) (2004) 1890-1893.

[167] J. Kong, N. R. Franklin, C. Zhou, M. G. Chapline, S. Peng, K. Cho, H. Dai, Nanotube molecular wires as chemical sensors, Science 287 (5453) (2000) 622-625.

[168] N. Elfström, R. Juhasz, I. Sychugov, T. Engfeldt, A. E. Karlström, J. Linnros, Surface charge sensitivity of silicon nanowires: Size dependence, Nano Lett. 7 (9) (2007) 2608-2612.

[169] C. R. Leao, A. Fazzio, A. J. da Silva, Si nanowires as sensors: Choosing the right surface, Nano letters 7 (5) (2007) 1172-1177.

[170] Z. Li, B. Rajendran, T. Kamins, X. Li, Y. Chen, R. S. Williams, Silicon nanowires for sequence-specific dna sensing: device fabrication and simulation, Applied Physics A 80 (6) (2005) 1257-1263.

[171] P. E. Sheehan, L. J. Whitman, Detection limits for nanoscale biosensors, Nano letters 5 (4) (2005) 803-807.

[172] J. Rife, M. Miller, P. Sheehan, C. Tamanaha, M. Tondra, L. Whitman, Design and performance of gmr sensors for the detection of magnetic microbeads in biosensors, Sensors and Actuators A: Physical 107 (3) (2003) 209-218. 
[173] P. R. Nair, M. A. Alam, Screening-limited response of nanobiosensors, Nano letters 8 (5) (2008) 1281-1285.

[174] F. Patolsky, G. Zheng, O. Hayden, M. Lakadamyali, X. Zhuang, C. M. Lieber, Electrical detection of single viruses, Proc. Natl. Acad. Sci. USA 101 (39) (2004) 14017-14022.

[175] A. A. Talin, L. L. Hunter, F. Léonard, B. Rokad, Large area, dense silicon nanowire array chemical sensors, Applied physics letters 89 (15) (2006) 153102.

[176] O. Elibol, D. Morisette, D. Akin, J. Denton, R. Bashir, Integrated nanoscale silicon sensors using top-down fabrication, Applied Physics Letters 83 (22) (2003) 4613-4615.

[177] M. C. McAlpine, H. Ahmad, D. Wang, J. R. Heath, Highly ordered nanowire arrays on plastic substrates for ultrasensitive flexible chemical sensors, Nature materials 6 (5) (2007) 379-384.

[178] Z. Li, Y. Chen, X. Li, T. Kamins, K. Nauka, R. S. Williams, Sequencespecific label-free dna sensors based on silicon nanowires, Nano Letters 4 (2) (2004) 245-247.

[179] J.-i. Hahm, C. M. Lieber, Direct ultrasensitive electrical detection of dna and dna sequence variations using nanowire nanosensors, Nano Lett. 4 (1) (2004) 51-54.

[180] N. N. Mishra, W. C. Maki, E. Cameron, R. Nelson, P. Winterrowd, S. K. Rastogi, B. Filanoski, G. K. Maki, Ultra-sensitive detection of bacterial toxin with silicon nanowire transistor, Lab Chip 8 (6) (2008) 868-871.

[181] E. Stern, R. Wagner, F. J. Sigworth, R. Breaker, T. M. Fahmy, M. A. Reed, Importance of the debye screening length on nanowire field effect transistor sensors, Nano letters 7 (11) (2007) 3405-3409. 
[182] X. Zhou, J. Hu, C. Li, D. Ma, C. Lee, S. Lee, Silicon nanowires as chemical

[183] W. Chen, H. Yao, C. H. Tzang, J. Zhu, M. Yang, S.-T. Lee, Silicon nanowires for high-sensitivity glucose detection, Appl. Phys. Lett. 88 (21) (2006) 213104.

[184] M.-W. Shao, Y.-Y. Shan, N.-B. Wong, S.-T. Lee, Silicon nanowire sensors

[187] K. Kneipp, Y. Wang, H. Kneipp, L. T. Perelman, I. Itzkan, R. R. Dasari, M. S. Feld, Single molecule detection using surface-enhanced raman scattering (sers), Phys. Rev. Lett. 78 (9) (1997) 1667.

[188] D. L. Jeanmaire, R. P. Van Duyne, Surface raman spectroelectrochemistry: Part i. heterocyclic, aromatic, and aliphatic amines adsorbed on the anodized silver electrode, J. Electroanal. Chem. 84 (1) (1977) 1-20.

[189] X. Han, H. Wang, X. Ou, X. Zhang, Highly sensitive, reproducible, and stable sers sensors based on well-controlled silver nanoparticle-decorated silicon nanowire building blocks, Journal of materials chemistry 22 (28) (2012) 14127-14132.

[190] Y. C. Cao, R. Jin, C. A. Mirkin, Nanoparticles with raman spectroscopic fingerprints for dna and rna detection, Science 297 (5586) (2002) 15361540.

[191] S. Schlücker, Surface-enhanced raman spectroscopy: Concepts and chem1355 ical applications, Angew. Chem. Int. Edit. 53 (19) (2014) 4756-4795. 
[192] M.-L. Zhang, C.-Q. Yi, X. Fan, K.-Q. Peng, N.-B. Wong, M.-S. Yang, R.Q. Zhang, S.-T. Lee, A surface-enhanced raman spectroscopy substrate for highly sensitive label-free immunoassay, Applied physics letters 92 (4) (2008) 43116-43116.

[200] M.-W. Shao, M.-L. Zhang, N.-B. Wong, D. D.-d. Ma, H. Wang, W. Chen, S.-T. Lee, Ag-modified silicon nanowires substrate for ultrasensitive 
surface-enhanced raman spectroscopy, Appl. Phys. Lett. 93 (23) (2008) 233118.

[206] R. Miao, L. Mu, H. Zhang, G. She, B. Zhou, H. Xu, P. Wang, W. Shi, Silicon nanowire-based fluorescent nanosensor for complexed cu2+ and its bioapplications, Nano Lett. 14 (6) (2014) 3124-3129.

[207] D. J. Maxwell, J. R. Taylor, S. Nie, Self-assembled nanoparticle probes for recognition and detection of biomolecules, J. Am. Chem. Soc. 124 (32) (2002) 9606-9612.

[208] R. Miao, L. Mu, H. Zhang, H. Xu, G. She, P. Wang, W. Shi, Modified silicon nanowires: a fluorescent nitric oxide biosensor with enhanced selectivity and stability, J. Mater. Chem. 22 (8) (2012) 3348-3353. 

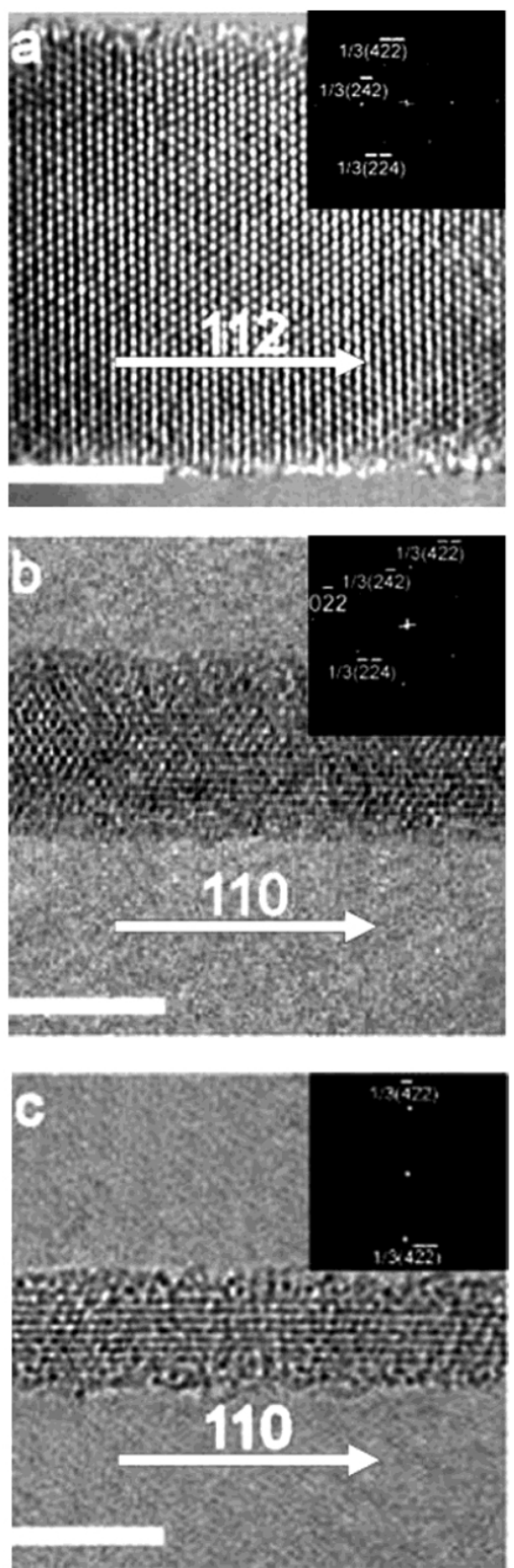

Figure 1: HRTEM images of Si NWs with diameters of (a) $13.2 \mathrm{~nm}$, (b) $5.7 \mathrm{~nm}$ and (c) $3.5 \mathrm{~nm}$. The three wires correspond respectively to $\langle 112\rangle,\langle 110\rangle$, and $\langle 111\rangle$ crystallographic orientations. Reprinted with permission from ref [17]. 


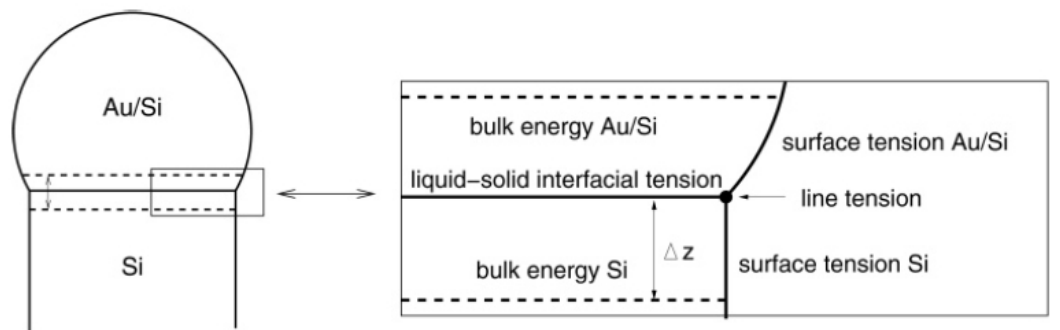

Figure 2: Solid-liquid interface region during Vapor-Liquid-Solid (VLS) growth process. Reprinted with permission from ref 63 .

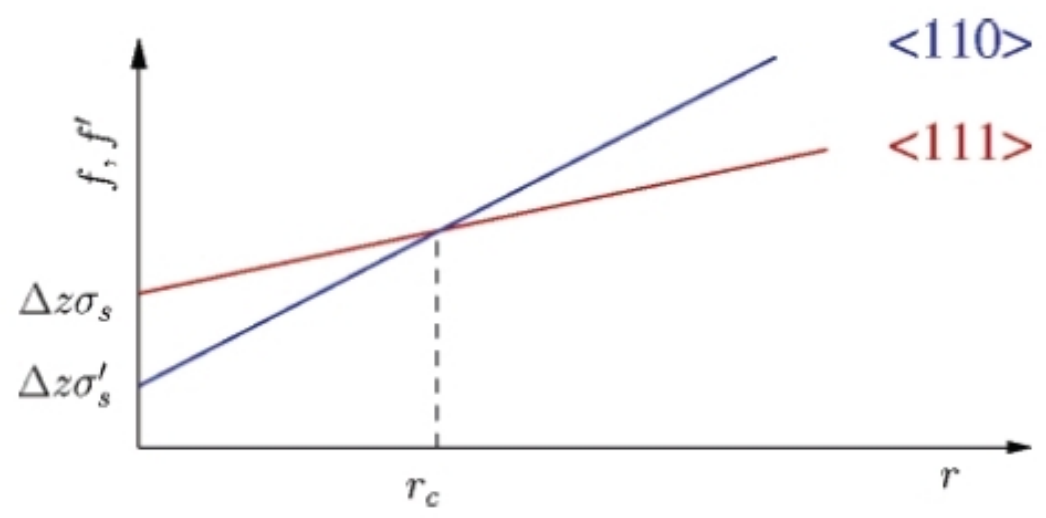

Figure 3: Specific free energy plots for $\langle 111\rangle$ (red line) and $\langle 110\rangle$ (blue line) oriented nanowires as a function of the radius $r$. Reprinted with permission from ref 63 . 


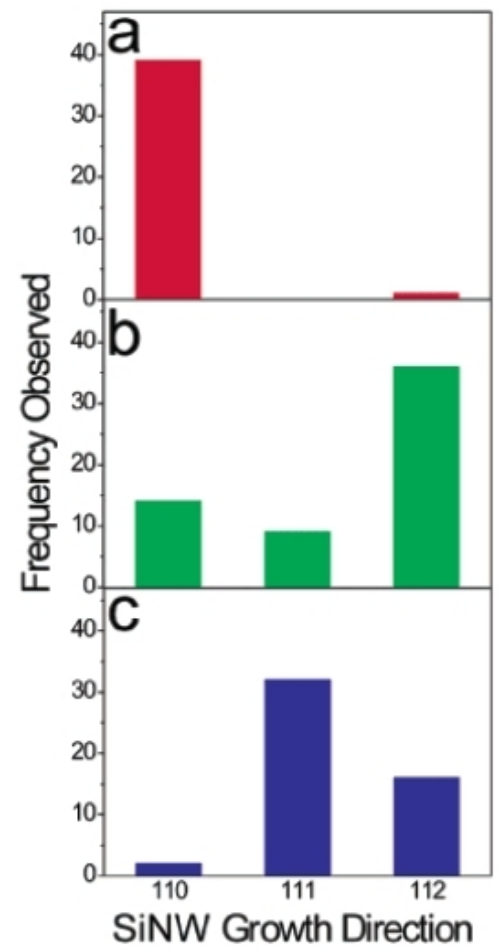

Figure 4: Distribution of samples with a specific growth direction for the three class of diameters: (a) 3 to $10 \mathrm{~nm}$, (b) 10 to $20 \mathrm{~nm}$, (c) 20 to $30 \mathrm{~nm}$. Reprinted with permission from ref [17. 

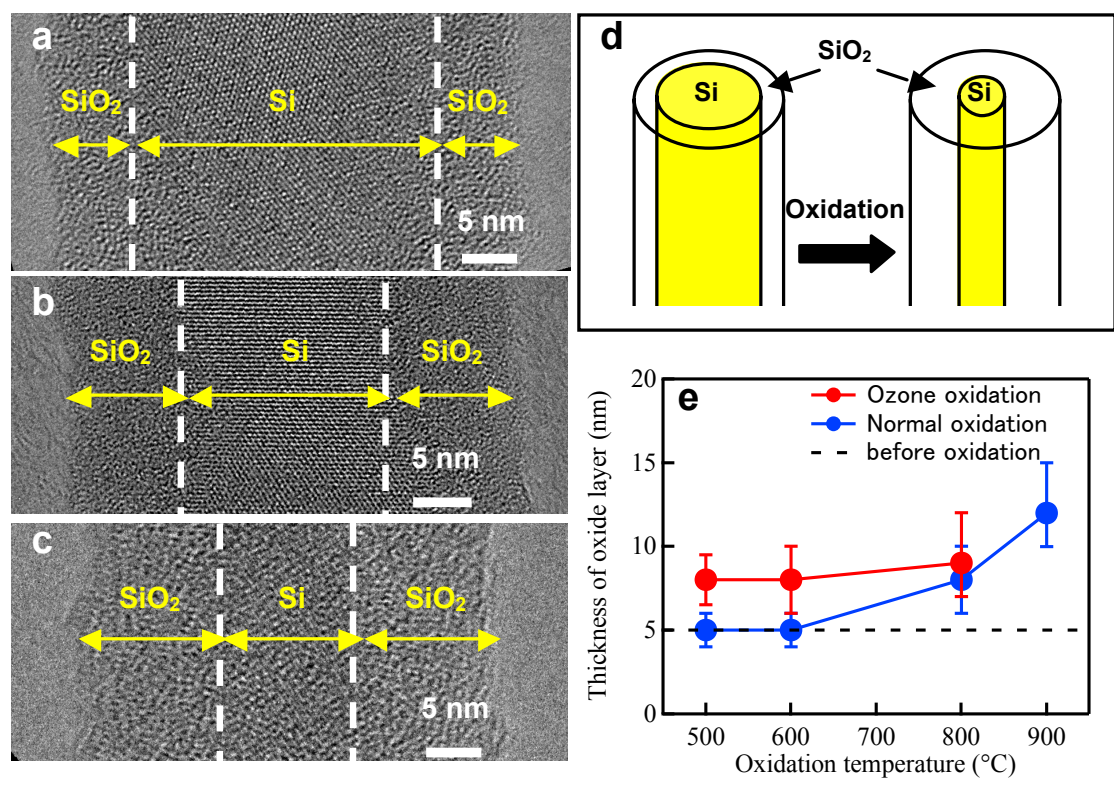

Figure 5: High-resolution images of a SiNWs (a) before oxidation and after (b) ozone oxidation at $600^{\circ} \mathrm{C}$ and (c) normal oxidation at $600^{\circ} \mathrm{C}$. The oxidation times are $180 \mathrm{~min}$. (d) A schematic illustration of thermal oxidation of SiNWs. (e) Dependence of the thickness of the surface oxide layer on oxidation temperature. Reprinted with permission from ref [20]. 


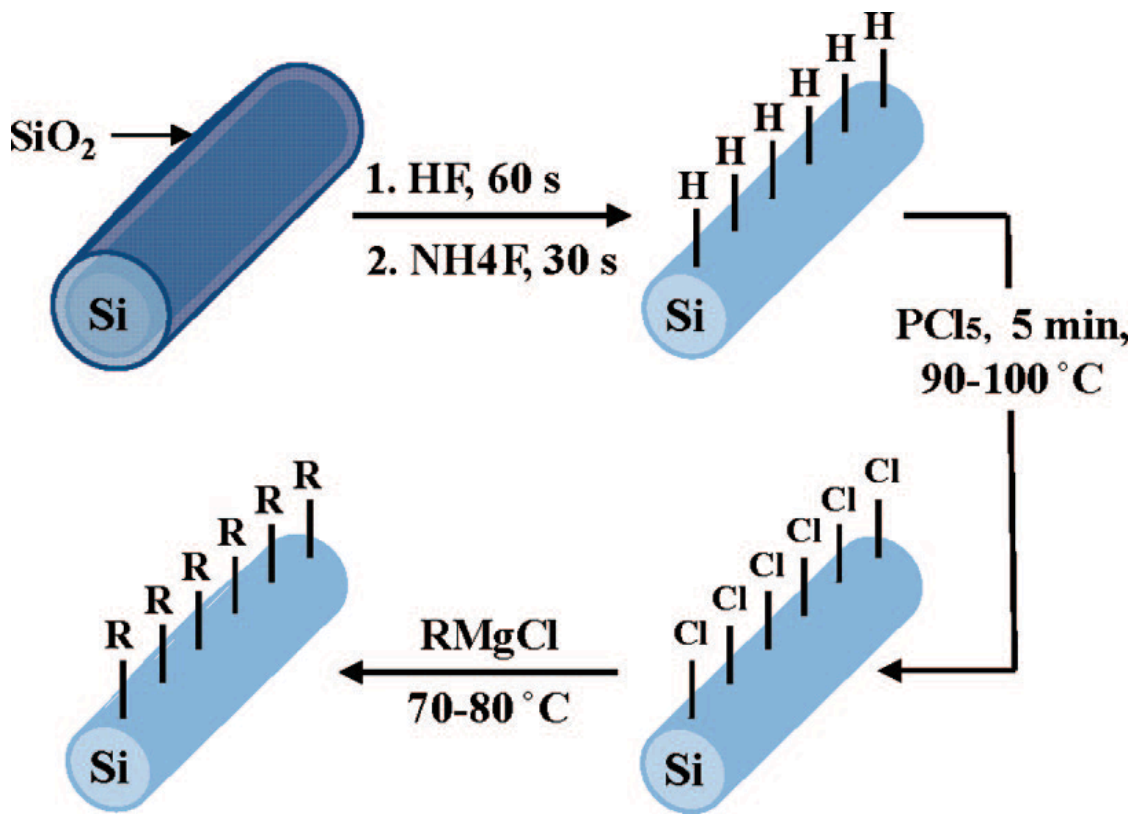

Figure 6: Sketch of the functionalization of Si NWs through the two step chlorination/alkylation process. Reprinted with permission from ref [86]. 


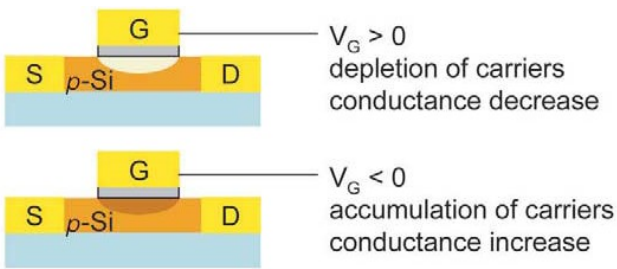

Figure 7: Side view of a traditional 2D FET device. Letters S, D and G stand for source, drain and gate. Reprinted with permission from ref [144]. 


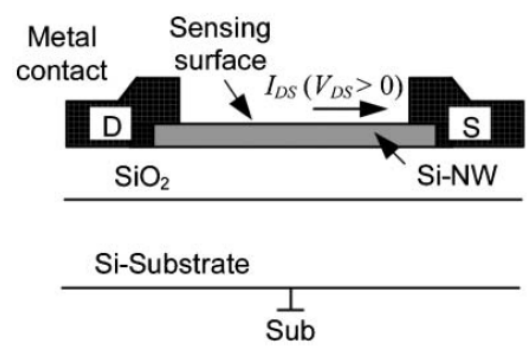

Figure 8: Schematic view of a Si NW cross section, in which the drain (D), the source (S) and the electrical current $\left(\mathrm{I}_{D S}\right)$ are shown. The graph is not in scale. Reprinted with permission from ref [147. 

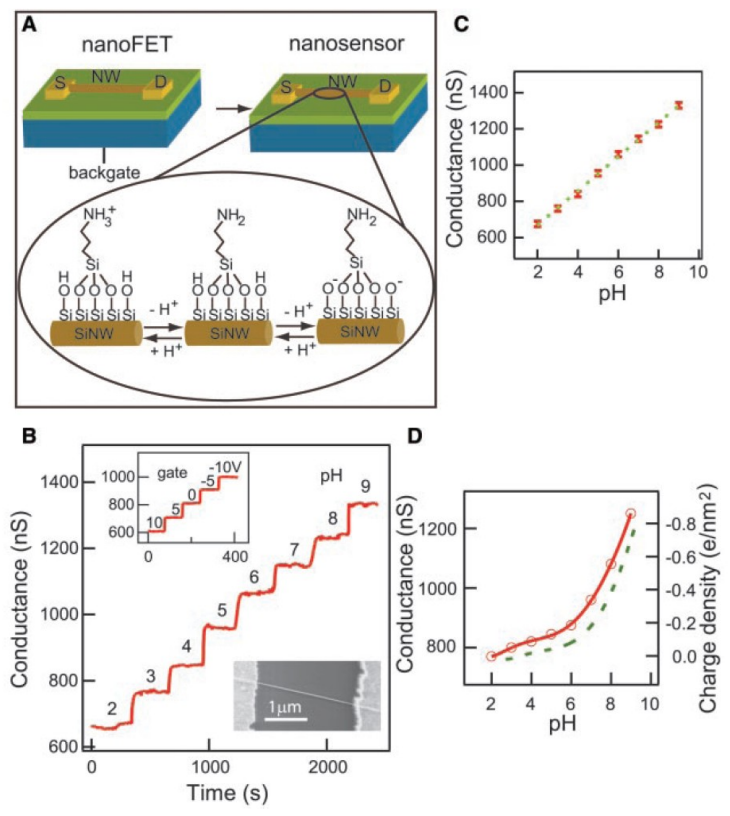

Figure 9: First Si NW based FET device for chemical and biological detection. (A) The steps of the conversion of a NW FET into NW nanosensors for $\mathrm{pH}$ sensing are shown. The NW is contacted with two electrodes, a source (S) and drain (D), for measuring conductance. Zoom of the APTES-modified SiNW surface showing the effect of $\mathrm{pH}$ in the surface charge state. (B) The real-time measurements of the conductance for an APTES-modified SiNW for pHs from 2 to 9; the $\mathrm{pH}$ values are indicated on the conductance plot. (Inset, top) Plot of the time-dependent conductance of a SiNW FET as a function of the back-gate voltage. (Inset, bottom) Field-emission scanning electron microscopy image of a typical SiNW device. (C) Dependence of the conductance on the $\mathrm{pH}$ level; the red points are experimental data, and the dashed green line is a linear fit through this data. (D) The conductance of unmodified SiNW (red) as a function of $\mathrm{pH}$. The dashed green curve is a plot of the surface charge density for silica as a function of $\mathrm{pH}$. Reprinted with permission from ref 143 . 


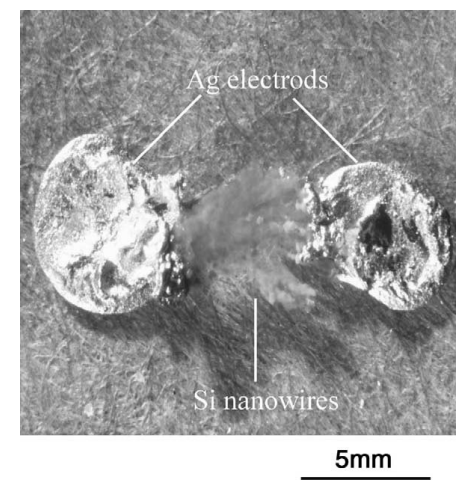

Figure 10: Optical micrograph of a Si NW connected to the two Ag electrodes. Reprinted with permission from ref 182 . 

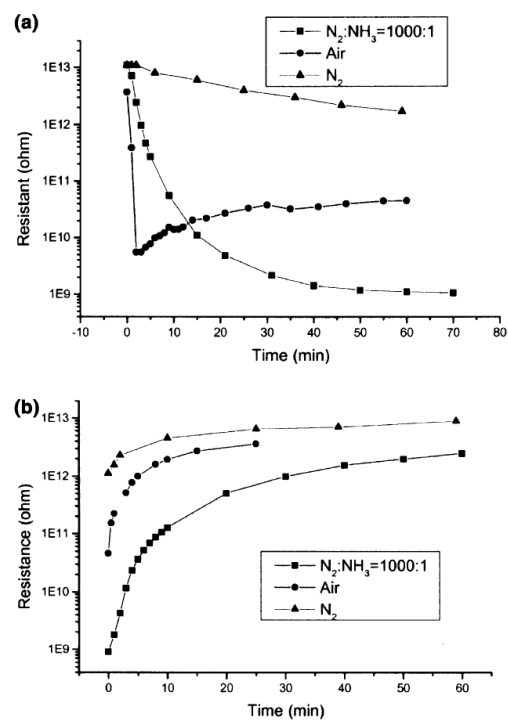

Figure 11: Resistance measurement of a Si NW exposed to $\mathrm{N}_{2}$, a mixture of $\mathrm{N}_{2}$ and $\mathrm{NH}_{3}$ and air with a relative humidity of $60 \%$. Top panel refers to the introduction of the gases into the chamber while bottom report the measurements done when the gas are pumped away. Reprinted with permission from ref 182 . 


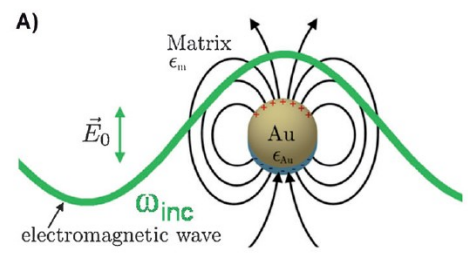

B)

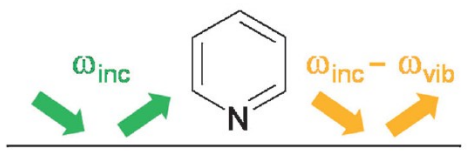

LSPR-supporting metal nanostructure

Figure 12: Underlying physics of SERS. (A) The incoming electromagnetic wave of frequency $\omega_{\text {inc }}$ can excite delocalized electrons in the metal of dielectric function $\epsilon_{A u}$ in a surrounding medium with dielectric function $\epsilon_{M}$. (B) Enhancement of incoming and out coming fields due to the interaction with the LSPR of the metal nanostructures. Reprinted with permission from ref [191]. 


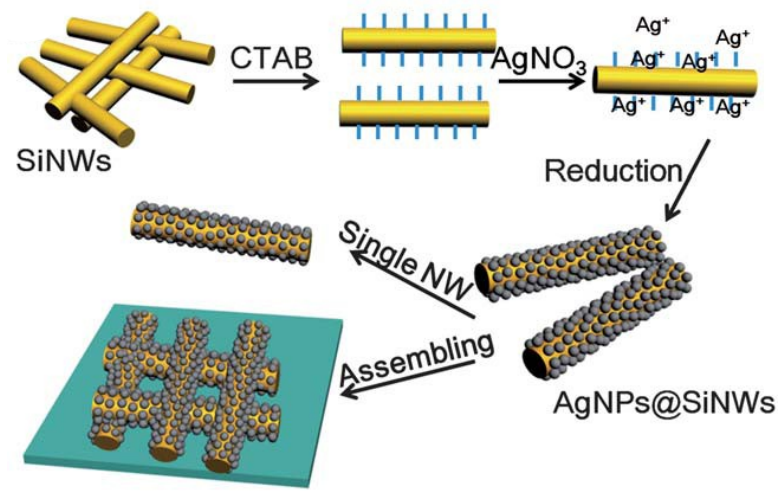

Figure 13: Schematic of the realization of Ag NPs functionalized Si NWs in solution for SERS substrates. The metal ions reduction' reaction is highlighted. Reprinted with permission from ref [189. 
(b)
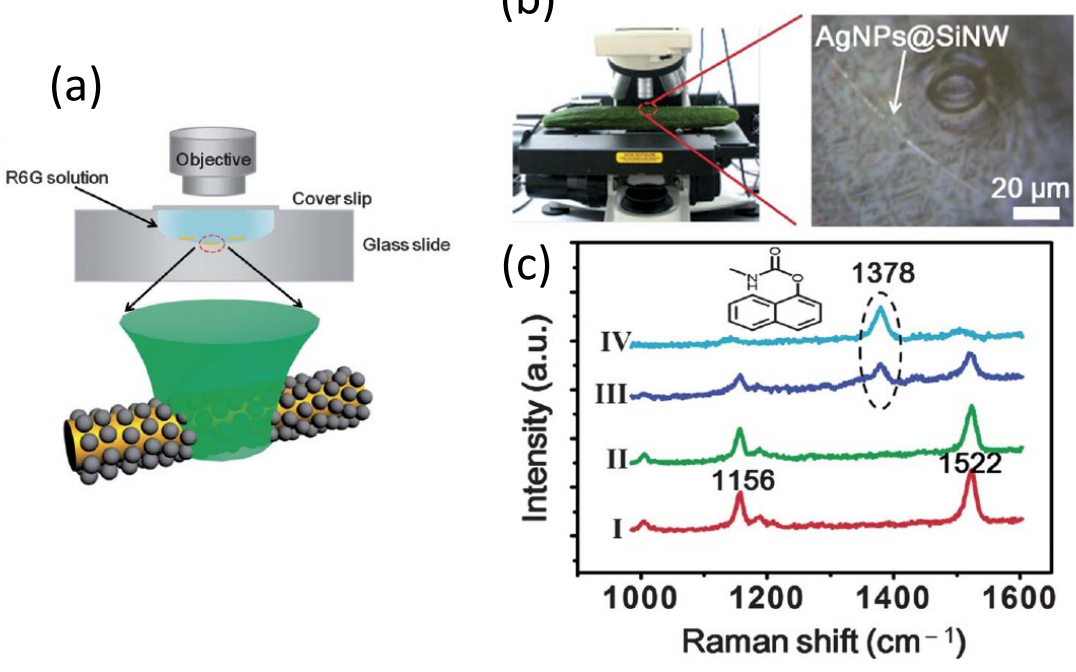

Figure 14: (a) Schematic of an AgNPs@SiNWs SERS single wire substrate. (b) Photograph of the detection of the pesticide residue on a cucumber surface experiment (left) and the microscope image of a single AgNP@SiNW transferred to the rough cucumber surface (right). (c) Recorded Raman spectra for clean cucumber surface (curve I), carbaryl contaminated surface (curve II), SERS spectra of a carbaryl contaminated surface modified by a single AgNP@SiNW (curve III), SERS spectra of pure carbaryl (curve IV). The presence of carbaryl can be easily recognized looking at the peak at $1378 \mathrm{~cm}^{-1}$ in curve III and IV appearing after transferring the AgNPs@SiNWs onto the probed surface. Reprinted with permission from ref 189 . 